\title{
Cyclin D1 overexpression induces global transcriptional downregulation in lymphoid neoplasms
}

\author{
Robert Albero, ${ }^{1}$ Anna Enjuanes, ${ }^{2,3}$ Santiago Demajo, ${ }^{1}$ Ciancarlo Castellano, ${ }^{4}$ Magda Pinyol,, 3 Noelia García, ${ }^{2}$ Cristina Capdevila, \\ Guillem Clot, ${ }^{1}$ Helena Suárez-Cisneros, ${ }^{2}$ Mariko Shimada, ${ }^{5,6}$ Kennosuke Karube, ${ }^{5,6}$ Mónica López-Guerra, ${ }^{1,3,6}$ Dolors Colomer, ${ }^{1,3,6}$ \\ Sílvia Beà, ${ }^{1,3}$ José Ignacio Martin-Subero, ${ }^{1,3}$ Elías Campo, ${ }^{1,3,6}$ and Pedro Jares s,3,4,6 $^{1,3}$ \\ `Lymphoid Neoplasm Program and ²Cenomics Unit, August Pi i Sunyer Biomedical Research Institute (IDIBAPS), Barcelona, Spain. ${ }^{3}$ Centro de Investigación Biomédica en Red de Cáncer (CIBERONC), \\ Barcelona, Spain. " Molecular Biology Core, Hospital Clinic of Barcelona, Barcelona, Spain. ${ }^{5}$ Hematopathology Unit and Cell Biology, Graduate School of Medicine and Faculty of Medicine, \\ University of the Ryukyus, Nishihara, Japan. ${ }^{6}$ Haematopathology Unit, Department of Anatomic Pathology, Hospital Clínic, University of Barcelona, Barcelona, Spain.
}

\begin{abstract}
Cyclin D1 is an oncogene frequently overexpressed in human cancers that has a dual function as cell cycle and transcriptional regulator, although the latter is widely unexplored. Here, we investigated the transcriptional role of cyclin D1 in lymphoid tumor cells with cyclin D1 oncogenic overexpression. Cyclin D1 showed widespread binding to the promoters of most actively transcribed genes, and the promoter occupancy positively correlated with the transcriptional output of targeted genes. Despite this association, the overexpression of cyclin D1 in lymphoid cells led to a global transcriptional downmodulation that was proportional to cyclin D1 levels. This cyclin D1-dependent global transcriptional downregulation was associated with a reduced nascent transcription and an accumulation of promoter-proximal paused RNA polymerase II (Pol II) that colocalized with cyclin D1. Concordantly, cyclin D1 overexpression promoted an increase in the Poll II pausing index. This transcriptional impairment seems to be mediated by the interaction of cyclin D1 with the transcription machinery. In addition, cyclin D1 overexpression sensitized cells to transcription inhibitors, revealing a synthetic lethality interaction that was also observed in primary mantle cell lymphoma cases. This finding of global transcriptional dysregulation expands the known functions of oncogenic cyclin D1 and suggests the therapeutic potential of targeting the transcriptional machinery in cyclin D1-overexpressing tumors.
\end{abstract}

\section{Introduction}

Cyclin D1 plays a central role in cell cycle regulation, and it is frequently upregulated in cancer by different genomic alterations, including amplifications in breast and respiratory tract tumors (1-4) and chromosomal translocations in mantle cell lymphoma (MCL) and multiple myeloma (MM) $(5,6)$. Moreover, point mutations disrupting the nuclear export process have been described in esophageal and endometrial carcinomas $(7,8)$. All these genetic alterations lead to an oncogenic overexpression of cyclin D1. Remarkably, CCND1, which encodes cyclin D1, was the most significantly amplified gene among the 12 tumor types recently analyzed in the Cancer Genome Atlas Pan-Cancer analysis project (9).

The classical tumorigenesis model considers that cyclin D1 mediates its oncogenic effect through its binding to CDK4, followed by $\mathrm{pRB}$ phosphorylation, E2F release, and subsequent promotion of the $G_{1} / S$ phase transition (10). However, during the last decade, a growing body of evidence has established that cyclin D1 has additional roles besides its canonical cell cycle function (1113). The description of cyclin D1 interactions with transcription factors and with chromatin-remodeling and histone-modifying

Authorship note: RA and $\mathrm{AE}$ contributed equally to this work. Conflict of interest: The authors have declared that no conflict of interest exists. Submitted: July 26, 2017; Accepted: June 28, 2018

Reference information: / Clin Invest. 2018;128(9):4132-4147.

https://doi.org/10.1172/JCI96520. enzymes has revealed a potential role of cyclin D1 as a transcriptional regulator in different cell models (14-17). However, whether oncogenic overexpression of cyclin D1 is responsible for transcriptional dysregulation in cancer cells remains unknown.

MCL is an aggressive lymphoid neoplasm that represents the paradigm of a neoplasia with cyclin D1-dependent oncogenesis. The t(11;14) (q13;q32) translocation that leads to constitutive overexpression of cyclin D1 is the initial oncogenic event in this lymphoma (18). The relevance of cyclin D1 dysregulation in MCL pathogenesis is stressed by the recognition that MCL cells adopt different mechanisms to increase the levels of cyclin D1, such as amplification of the translocated allele or secondary rearrangements and mutations involving the $3^{\prime}$ untranslated region that generate more stable CCND1 transcripts (19-21). The expression of these abnormal transcripts correlates with the presence of higher protein levels and increased aggressiveness of the tumors (22). Recently, mutations at the cyclin D1 N-terminal region have been identified in MCL that also lead to increased stability of the protein $(23,24)$.

In this study, we have investigated the role of cyclin D1 overexpression as a transcriptional regulator in malignant lymphoid cells. Integration of ChIP sequencing (ChIP-Seq) data on cyclin D1 with data on histone modifications and the transcriptional output of MCL cell lines revealed that cyclin D1 binds to the promoters of most actively transcribed genes, and its overexpression led to global downmodulation of the transcriptome program. This effect was associated with an accumulation of promoter-proximal 
paused RNA polymerase II (Pol II) that overlapped with cyclin D1-bound regions. In concordance with the presence of higher levels of paused Pol II, the overexpression of cyclin D1 promoted an increase in the Pol II pausing index. This transcriptional dysregulation seems to be mediated by the physical interaction of cyclin with the transcription machinery. Finally, cyclin D1-overexpressing cells showed greater sensitivity to transcription inhibitors, a phenotype also observed in primary MCL cases, suggesting a synthetic lethality interaction that may open new therapeutic opportunities in cyclin D1-overexpressing tumors.

\section{Results}

Cyclin D1 shows extensive genome-wide chromatin binding in $M C L$ cells. In order to characterize the genome-wide chromatin binding pattern of cyclin D1, we performed ChIP-Seq of endogenous cyclin D1 in 4 MCL cell lines (Z-138, GRANTA-519, Jeko-1, and UPN-1). All these cell lines carry the $\mathrm{t}(11 ; 14)$ translocation and display variable levels of cyclin D1 protein overexpression (Supplemental Figure 1A; supplemental material available online with this article; https://doi.org/10.1172/JCI96520DS1). Of note, we found a high number of cyclin D1 DNA-binding regions, with 19,860 peaks common to all $4 \mathrm{MCL}$ cell lines (Figure 1A). Interestingly, the number of identified peaks displayed a strong positive correlation with the amount of cyclin D1 protein $(r=0.87)$ (Supplemental Figure 1B). The annotation of the peaks as promoter, gene body (exon or intron), or intergenic revealed enrichment in promoters (Supplemental Table 1). Peaks at promoters showed higher tag density, and, concordantly, when a tag density filter was applied, more than $50 \%$ of the peaks were classified as promoters (Figure $1 \mathrm{~B}$ and Supplemental Table 2). In total, an average of 11,583 coding genes displayed cyclin D1 binding to their proximal promoters, and more than $74 \%$ of them were common among the 4 cell lines $(n=8,638)$ (Figure 1C). The actual distribution of cyclin D1-binding sites showed that these interactions tend to occur close to and centered around the transcription start sites (TSS) of the genes (Figure 1D). Functional pathway analysis of genes showing cyclin D1 occupancy at promoters revealed that these genes were related to processes such as translation, RNA processing, cell cycle, and DNA damage and repair, among others (Figure 1E and Supplemental Table 3).

To validate the cyclin D1 promoter binding, we performed ChIP-quantitative PCR (ChIP-qPCR) of 8 genes with cyclin D1 peaks close to their TSS (Figure $1 \mathrm{~F}$ and Supplemental Figure $1 \mathrm{C}$ ). All the tested genes showed marked cyclin D1 binding enrichment when compared with a negative region (Figure $1 \mathrm{G}$ ). The interaction of cyclin D1 with promoters is consistent with a transcriptional function of the cyclin in MCL cells, and the extensive binding observed across the genome is compatible with a global transcriptional role.

Cyclin D1 binds to open chromatin regions enriched in active histone marks. To further characterize the global binding of cyclin D1 and its potential transcriptional role, we investigated the features of the cyclin D1-associated chromatin. To do so, we compared the cyclin D1 binding pattern to the profiles of several histone marks and DNase I hypersensitive sites obtained for the Z-138 cell line in the context of the Blueprint Epigenome consortium (25). We observed that promoters occupied by cyclin D1 were enriched in active histone marks (H3K4me3 and H3K27ac) and DNase I hypersensitive sites (Figure 2, A and B). Conversely, cyclin D1 was not present at promoters lacking active histone marks and DNase I hypersensitive sites (Figure 2A). Although cyclin D1 localized preferentially to active promoters, characterized by the presence of H3K27ac and H3K4me3 histone modifications, a fraction of cyclin D1 peaks also occurred at active enhancers, characterized by H3K27ac and H3K4me1 histone modifications (Figure 2C). The cyclin D1 binding density at active promoters was significantly higher than that observed in enhancers (Figure 2D; $P<2.2 \times 10^{-16}$ ). Taken together, these results indicate that cyclin D1 binds to regions of open chromatin enriched in active histone marks, which include promoters and enhancers.

Cyclin D1 occupies promoters of highly expressed genes. To study the relationship between the binding of cyclin D1 to promoters and the transcriptional output, we performed RNA sequencing (RNA-Seq) of the MCL cell lines. Interestingly, when all coding genes were stratified according to mRNA expression levels, the groups with higher gene expression values displayed larger fractions of cyclin D1-bound genes (Figure 3A). Furthermore, cyclin D1 promoter occupancy showed a strong positive correlation with gene expression levels $\left(\rho=0.98, P<2.2 \times 10^{-16}\right)$ (Figure 3B), and the average binding density around the TSS was proportional to the transcription output of the genes (Figure 3C and Supplemental Figure 2A). These results were consistent with the high degree of overlap observed between cyclin D1-binding sites and active chromatin marks. Moreover, when we combined the expression of primary MCL cases $(n=122)$ with the cyclin D1 peak density found in MCL cell lines, we observed a highly significant correlation between cyclin D1 promoter binding density in MCL cell lines and expression levels of the genes in primary MCL cases (Figure $3 \mathrm{D}, \rho=0.97, P<2.2 \times 10^{-16}$ ). This result shows that genes actively transcribed in MCL cell lines with cyclin D1 promoter binding are also highly expressed in primary MCL cases. Together, our results demonstrate that cyclin D1 binding occurs predominantly around the TSS of abundantly transcribed genes, suggesting that cyclin D1 might regulate transcription in MCL cells.

Genome-wide recruitment of exogenous cyclin D1 to active gene promoters. We wanted to investigate whether exogenous cyclin D1 would be recruited to promoters and preferentially to the actively transcribed genes, in a manner similar to endogenous cyclin D1. To that end, we transduced a cyclin D1-negative lymphoblastoid leukemic cell line (JVM13) to constitutively express a nuclear active form of cyclin D1 carrying the T286A mutation (JVM13$\left.\mathrm{CD}^{\mathrm{T} 286 \mathrm{~A}}\right)$. This mutation prevents physiological nuclear export and subsequent degradation of the cyclin, allowing cyclin D1 protein to reach levels more similar to those observed in MCL cell lines (Supplemental Figure 2B). The cyclin D1 DNA binding profile observed in JVM13-cD1 $1^{\mathrm{T} 286 \mathrm{~A}}$ cells was comparable to the pattern identified in the MCL cell lines (Figure 3E), despite the fact that a lower number of cyclin D1 peaks was observed. The reduced number of peaks could be due to the lower amount of cyclin D1 protein constitutively expressed by JVM13-cD1 ${ }^{\mathrm{T} 286 \mathrm{~A}}$ compared with the MCL cell lines. In fact, the correlation observed between the number of identified peaks and the amount of cyclin D1 protein in MCL cell lines was strengthened when JVM13-cD1 ${ }^{\mathrm{T} 286 \mathrm{~A}}$ data were included ( $r=0.98$; Supplemental Figure 2C). This result suggests that the degree of cyclin D1 chromatin binding is proportional to the amount of cyclin D1 protein. 
A
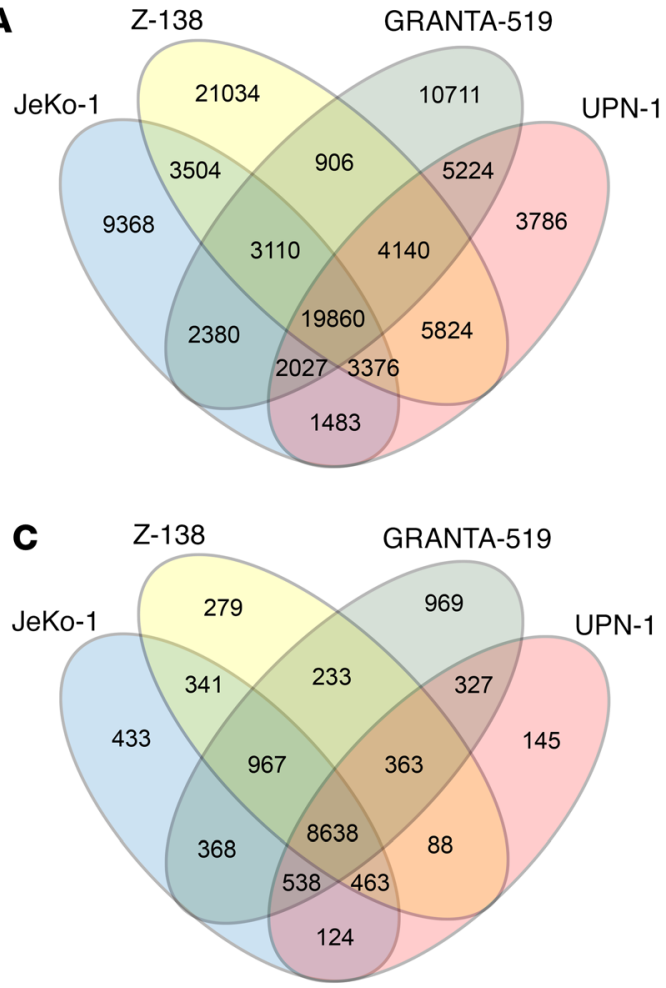

$\mathbf{E}$

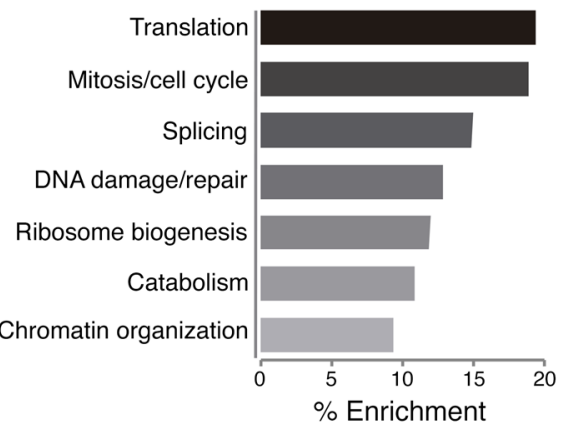

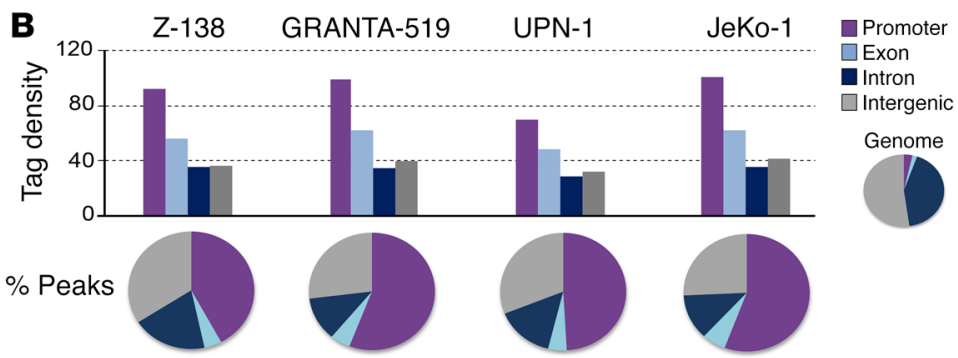

D
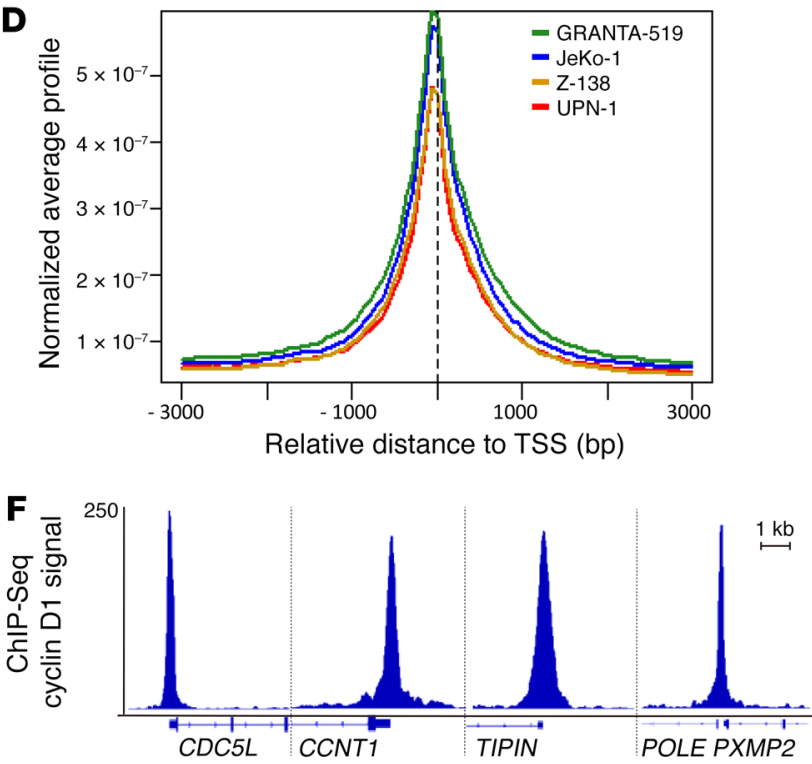

G

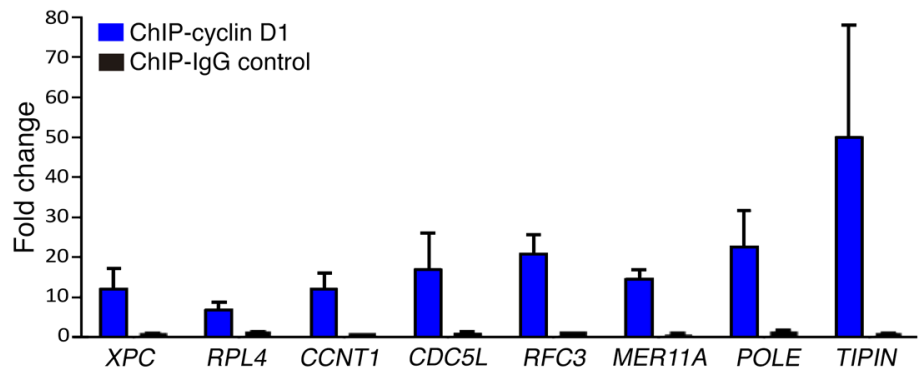

Figure 1. Cyclin D1 binds genome-wide in MCL cell lines. (A) Venn diagram representing cyclin D1 ChIP-Seq peaks in 4 MCL cell lines. (B) Distribution of cyclin D1-interacting regions over specific genomic regions in MCL cell lines. Box plots showing cyclin D1 tag density of the different genomic regions and pie charts displaying the genomic distribution of genomic intervals, with a number of tags higher than the mean. The distribution across the human genome is represented as a control. (C) Venn diagram representing cyclin D1-targeted genes identified by ChIP-Seq in MCL cell lines. Genes were considered targets when they displayed cyclin D1-binding sites located within $1 \mathrm{~kb}$ upstream of their TSS. (D) Average signal profile of cyclin D1 around the TSS $( \pm 3 \mathrm{~kb})$ in MCL cell lines. (E) Top hits of the functional annotation clustering analysis of common cyclin D1 target genes among the $4 \mathrm{MCL}$ cell lines. Only the genes with the most significant peaks in their promoters $(-\log P>350)$ were considered for the analysis. (F) Genome browser view of the ChIP-Seq tag density plots of 4 representative cyclin D1 target genes. (G) ChIP-qPCR validation of 8 selected cyclin D1 target genes in GRANTA-519. The fold change enrichments relative to a negative region are presented (mean $\pm \operatorname{SEM})(n=2)$.

To determine the possible association between the chromatin binding of exogenous cyclin D1 and the transcriptome output, we performed RNA-Seq of JVM13-cD1 $1^{\text {T286A }}$ cells. The integration of ChIP-Seq and RNA-Seq data sets showed a strong positive correlation between gene expression levels and exogenous cyclin D1 promoter density $\left(\rho=0.97, P<2.2 \times 10^{-16}\right.$; Figure $\left.3 F\right)$. Moreover, the average binding density around the TSS was proportional to the transcription levels of the genes, similar to what was observed for endogenous cyclin D1 (Supplemental Figure 2D). In addition, the combination of the basal gene expression profile of JVM13 cells with cyclin D1 chromatin binding showed that genes bound by exogenous cyclin D1 were already actively transcribed prior to the presence of cyclin D1, and the genome-wide recruitment around the TSS correlated with the previous mRNA levels of the 
A
H3K27ac H3K4me3 H3K4me1

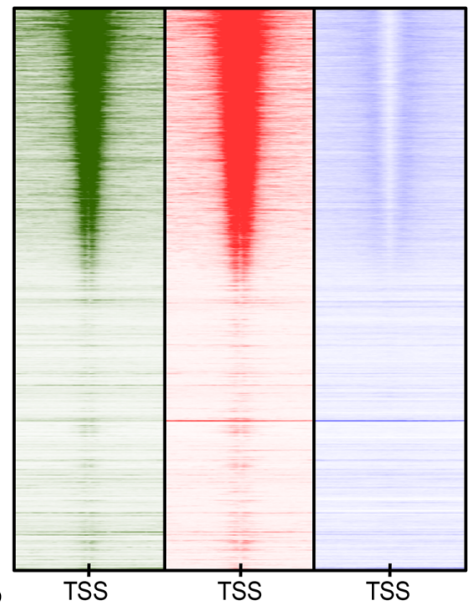

DNase

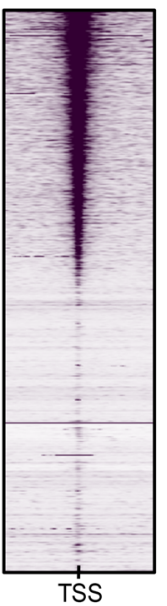

B
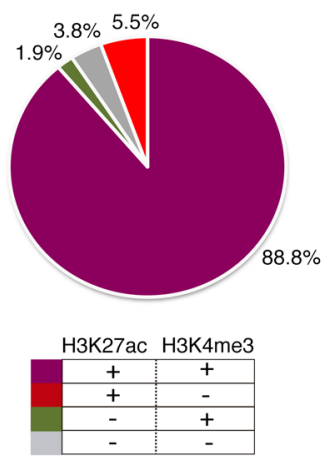

C

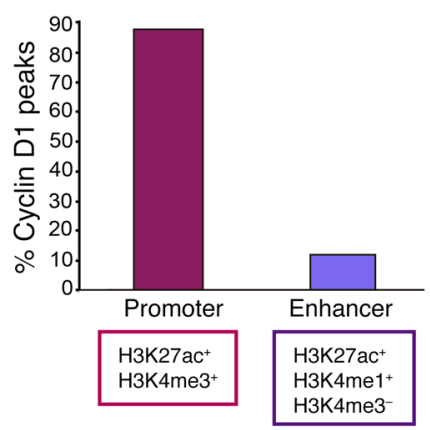

D

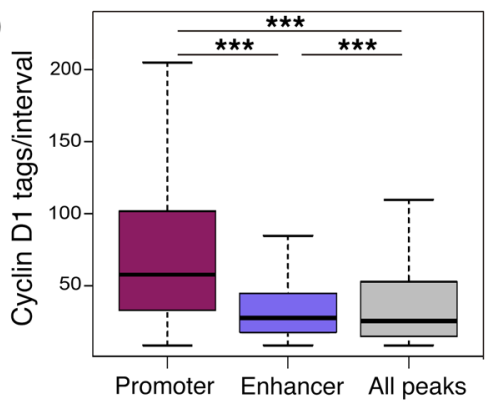

Figure 2. Cyclin D1 occupancy correlates with active promoter marks and open chromatin conformation. (A) Heatmap showing the ChIP-Seq tag density of cyclin D1, H3K27ac, H3K4me3, H3K4me1, and DNase I cutting sites around all genomic TSS in Z-138 cells. Each row represents a gene centered on the TSS ( $\pm 5 \mathrm{~kb}$ ). Promoters are sorted by cyclin D1 number of tags. Cyclin D1-bound (top) and -unbound (bottom) genes are shown. (B) Pie chart representing common regions bound by cyclin D1, H3K27ac, and H3K4me3 marks. Only cyclin D1 peaks at promoters (-5 kb TSS) in Z-138 cells are shown. (C) Cyclin D1 occupancy in active promoters and enhancers. Percentage of active promoters ( $\left.\mathrm{H} 3 \mathrm{~K} 4 \mathrm{me} 3^{+}\right)$and enhancers ( $\left.\mathrm{H} 3 \mathrm{~K} 4 \mathrm{me} 1^{+}, \mathrm{H} 3 \mathrm{~K} 4 \mathrm{me} 3^{-}\right)$colocalizing with cyclin D1 in active regions (defined by H3K27ac presence) are shown. (D) Box plot showing cyclin D1 number of tags in active promoters and active enhancers. The number of all cyclin D1 peaks is represented as control. ${ }^{* *} P<2.2 \times 10^{-16}$, Student's $t$ test, Holm-Bonferroni correction.

bound genes (Supplemental Figure 2E). Together, these results suggest that cyclin D1 may be recruited to promoters that are preconfigured in an active state, and the extent of recruitment would be dependent on the transcriptional levels of the targeted genes.

Cyclin D1 overexpression leads to a global reduction in RNA synthesis in lymphoid cells. The cyclin D1 chromatin binding pattern identified in MCL cell lines and JVM13 cells overexpressing cyclin D1 evidenced important similarities to that recently reported for MYC (26-28). Both proteins display extensive genome-wide binding to open chromatin, preferentially centered around the TSS of highly expressed genes, together with a unimodal distribution in a peak-density histogram (Supplemental Figure 3A and refs. 26-28). Indeed, when we compared the chromatin binding of cyclin D1 with the MYC peaks previously described in a normal B cell (GM12878), we observed that more than 23\% of promoters bound by cyclin $\mathrm{D} 1$ also had been reported to be occupied by MYC in normal B cells (29). Moreover, the 2 proteins showed a similar chromatin binding profile (Supplemental Figure 3B). In addition, the cyclin D1 peaks that colocalized with MYC showed a significantly higher tag density $\left(P<2.2 \times 10^{-16}\right.$; Supplemental Figure 3C). Interestingly, the particular chromatin binding pattern of MYC was associated with an increase in total RNA and mRNA, which led to the identification of MYC as a global transcriptional amplifier (26-28). To analyze whether the genome-wide chromatin binding pattern of cyclin D1 is related to a potential role of the cyclin as a global transcriptional regulator, we explored the relationship between cyclin D1 expression and total cellular RNA content. We overexpressed in JVM13 cells either the highly stable nuclear form of cyclin D1 (JVM13-D1 ${ }^{\text {T286A) }}$ or the WT form of the protein (JVM13-D1). As expected, inducible JVM13-D1 $1^{\text {T286A }}$ cells showed higher cyclin D1 protein levels than JVM13-D1 cells (Figure 4A). Following cyclin D1 induction, we performed spectrophotometric analysis of the absolute levels of total RNA obtained from a fixed number of cells (Figure 4B). Unexpectedly, cyclin D1-overexpressing cells showed significantly lower amounts of total RNA per cell than JVM13 control cells (Figure 4B). In order to exclude that the reduction in the amount of total RNA could be related to the cell cycle distribution of the cells, we measured the amount of total RNA by flow cytometry using pyronin Y (RNA) and Hoechst (DNA) costaining. This approach confirmed that the significant decrease in the absolute levels of RNA content due to cyclin D1 overexpression was independent of the cell cycle phase, 
A

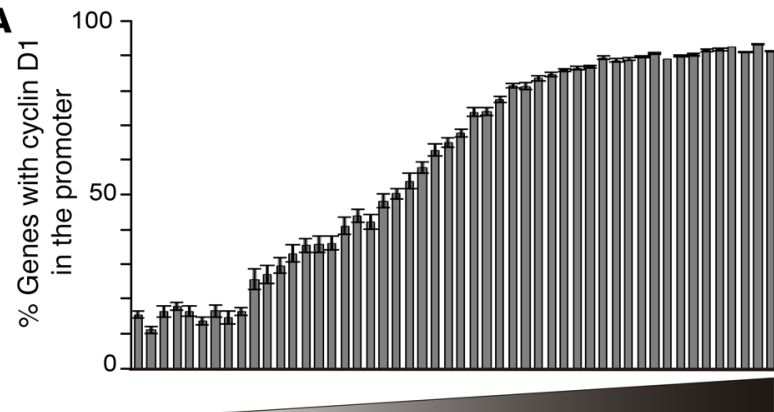

Gene expression levels

C

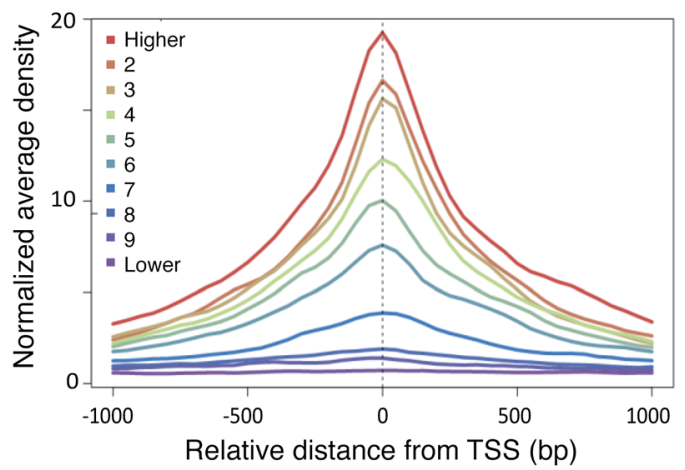

E

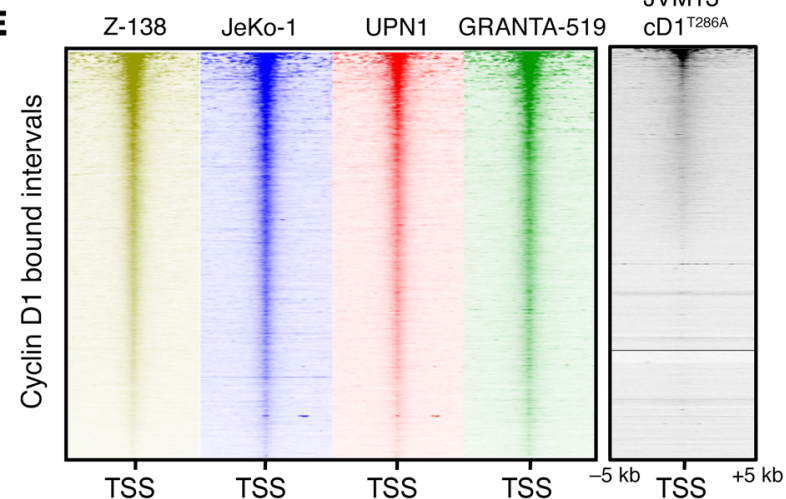

B

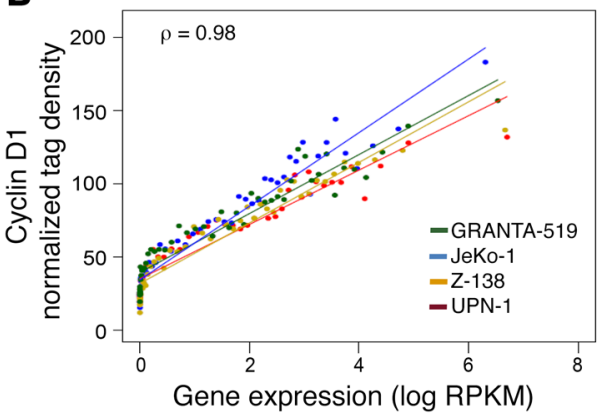

D

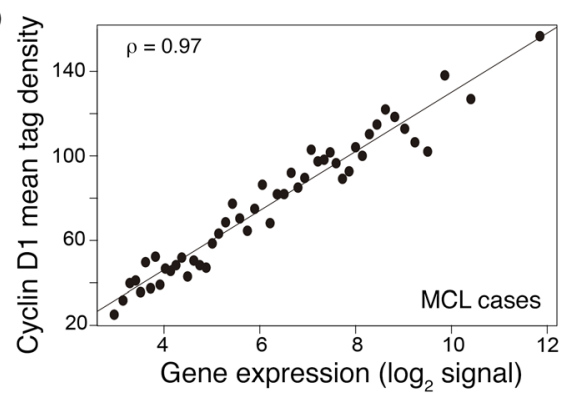

F

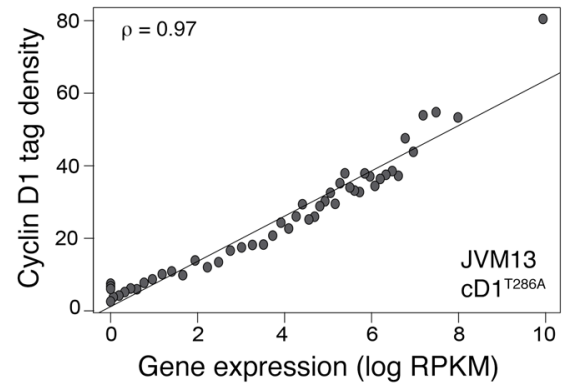

Figure 3. Cyclin D1 binding correlates with gene expression levels. (A) Distribution of genes showing cyclin D1 peaks within their promoters ( 5 kb upstream of the TSS) according to their respective gene expression levels. All genes were sorted into 50 equal bins based on their expression levels. Results are shown as mean \pm SEM of all 4 MCL cell lines. (B) Linear correlation between cyclin D1 binding and transcription. Genes were sorted as in A. The average of cyclin D1 ChIP-Seq normalized tag densities at promoters and the RPKM-normalized expression levels are shown for each bin. Spearman's correlation, $\rho=0.98, P<2.2 \times 10^{-16}$. (C) Profile of cyclin D1 occupancy around the TSS in Z-138 cells. Genes were divided into 10 groups based on their expression levels (from higher to lower expression). The distribution of the cyclin D1 ChIP-Seq tag density average around the TSS ( $\pm 1 \mathrm{~kb})$ is displayed for each group. (D) Linear correlation between cyclin D1 binding in MCL cell lines and gene expression in MCL primary samples ( $n=122$ ). Genes were sorted into 50 equal bins based on their expression in MCL samples. For each bin, the cyclin D1 ChIP-Seq tag density average in the MCL cell lines and the gene expression mean in primary samples are shown. Spearman's correlation, $\rho=0.97, P<2.2 \times 10^{-16}$. (E) Heatmap showing the cyclin D1 ChIP-Seq tag density within gene promoters of JVM13-cD1 ${ }^{\text {T286A }}$ and MCL cell lines. Each row represents a gene centered on the TSS ( $\left.\pm 5 \mathrm{~kb}\right)$. Promoters are sorted by the number of cyclin D1 tags in Z-138 cells. (F) Linear correlation between cyclin D1 binding and gene expression in JVM13-cD1 ${ }^{\mathrm{T} 286 \mathrm{~A}}$ cells. Genes were sorted into 50 equal bins as in B. Spearman's correlation, $\rho=0.97, P<2.2 \times 10^{-16}$.

since it was observed in both $\mathrm{G}_{1}$ and $\mathrm{G}_{2} / \mathrm{M}$ cells (Figure $4 \mathrm{C}$ and Supplemental Figure 4A). Remarkably, the reduction was greater in JVM13-D1 ${ }^{\mathrm{T} 286 \mathrm{~A}}$ cells, which express higher levels of cyclin D1 protein. In addition, we transduced the lymphoblastoid JVM2 cell line, which expresses low levels of endogenous cyclin D1 despite harboring the $\mathrm{t}(11 ; 14)$ translocation, with the stable nuclear (JVM2-D1 ${ }^{\mathrm{T} 286 \mathrm{~A}}$ ) or the WT form of cyclin D1 (JVM2-D1) (Supplemental Figure 4E). RNA quantification showed that JVM2-D1 ${ }^{\mathrm{T} 286 \mathrm{~A}}$ cells displayed a significant diminution in the amount of total RNA per cell when compared with JVM2 control cells. The reduction was not observed in JVM2-D1 cells, which displayed low cyclin D1 levels (Supplemental Figure 4, B, C, and E).

To further confirm these results, we knocked down cyclin D1 in the MCL cell line GRANTA-519 using 2 independent shRNA constructs (Figure 4D). In agreement with the overexpression experiments, cyclin D1 depletion led to a significant increase in the total 

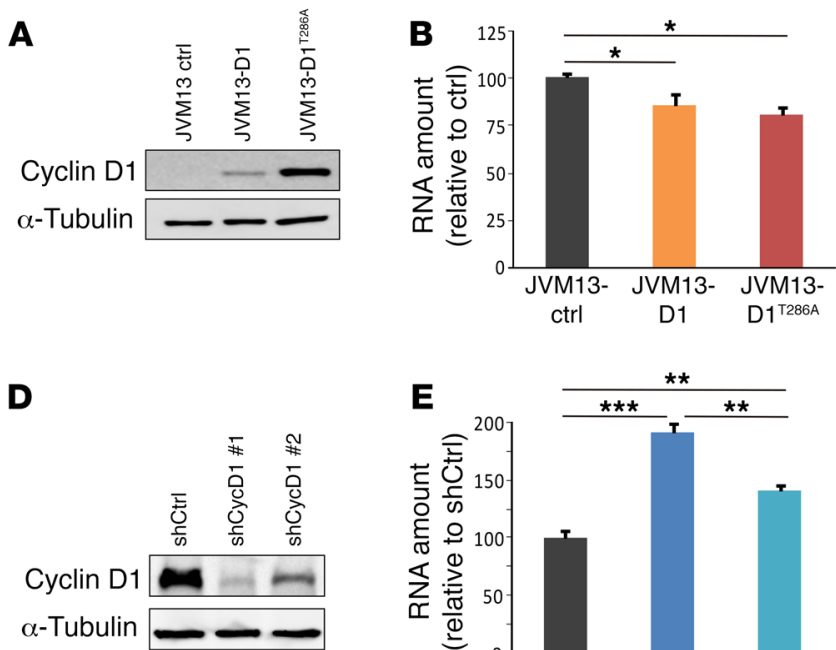
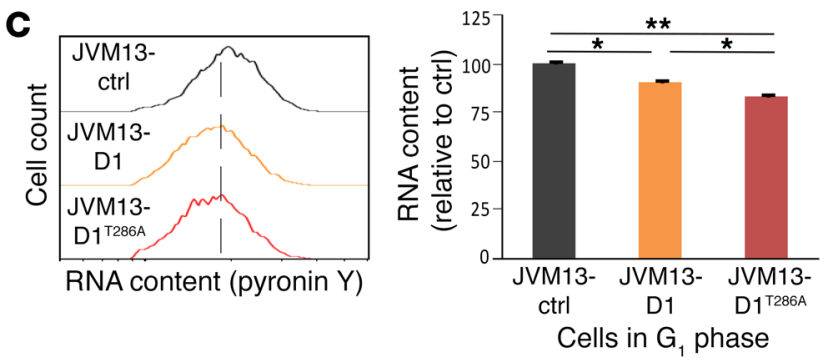

$\mathbf{F}$

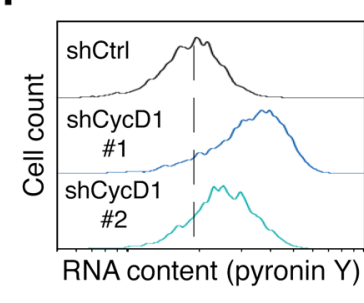

H
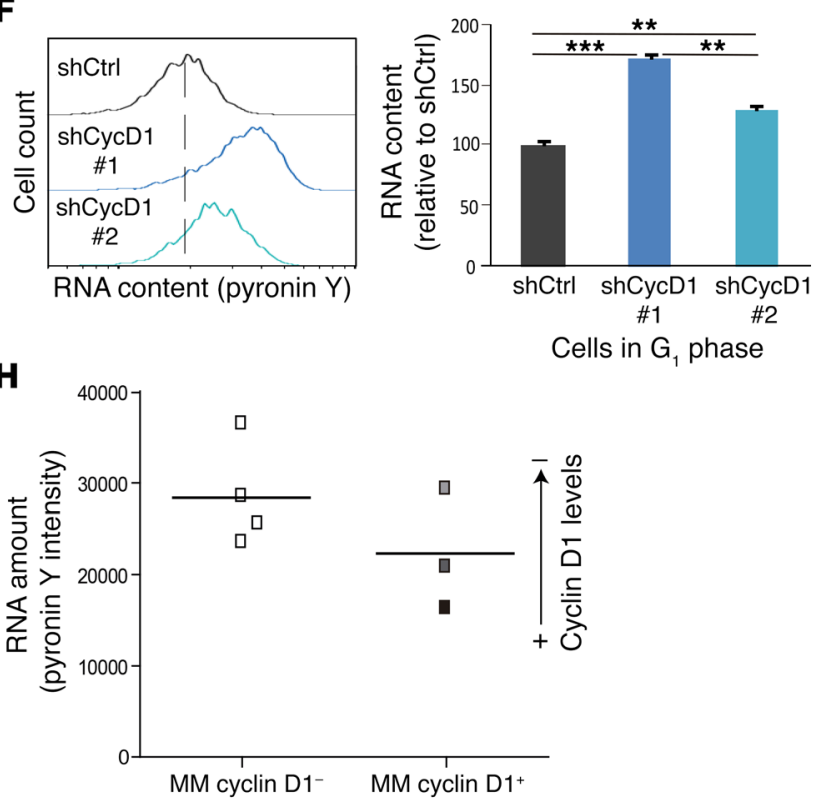

Cells in $G_{1}$ phase

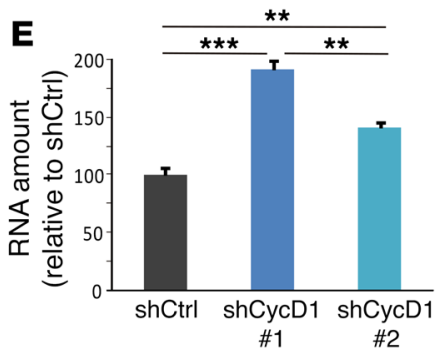

G

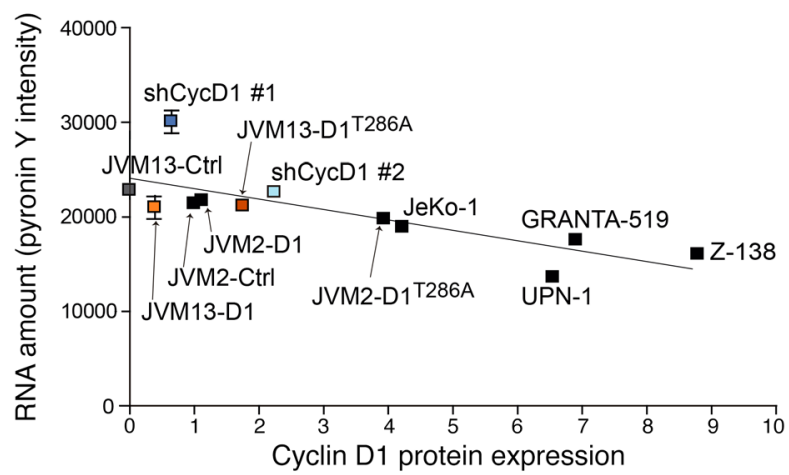

I

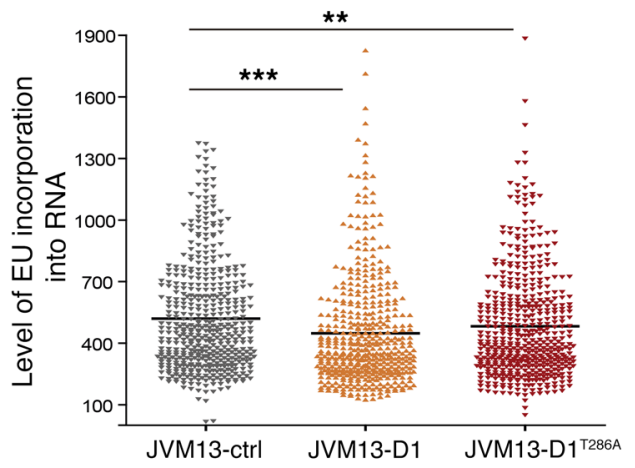

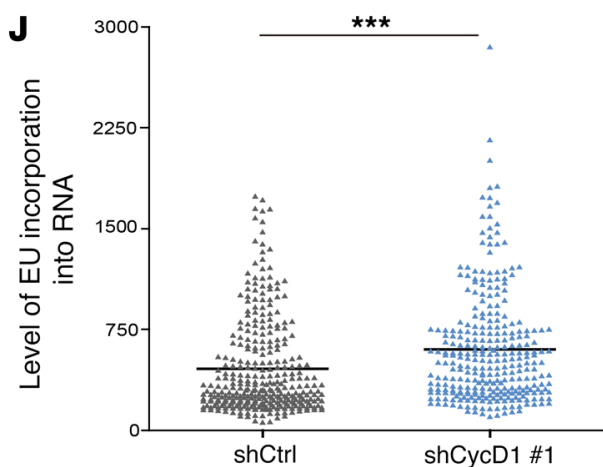

Figure 4. Cyclin D1 overexpression results in a reduction in the total RNA content in malignant lymphoid cells. (A) Cyclin D1 protein in JVM13-control (JVM13-ctrl), JVM13-D1, and JVM13-D1 ${ }^{\text {T286A }}$ cells. $\alpha$-Tubulin was used as loading control. (B) Total RNA content extracted from $10^{6}$ cells. Results are shown relative to the control (mean $\pm \mathrm{SEM}, n=9$ ). ${ }^{*} P<0.05$, Student's $t$ test. (C) RNA quantification by pyronin $Y$ staining in JVM13 inducible cell lines. Only cells in $G_{1}$ phase were analyzed. Left panel: FACS profile of a representative experiment. Right panel: Bar graph displaying the pyronin $Y$ mean signal. Results are shown relative to the control (mean $\pm \mathrm{SEM}, n=3$ ). ${ }^{*} P<0.05$, ${ }^{* *} P<0.01$, Student's $t$ test. (D) Cyclin D1 expression in control (shCtrl) and cyclin D1-depleted (shCycD1 \#1 and \#2) GRANTA-519 cells. $\alpha$-Tubulin was used as loading control. (E) Total RNA content in cyclin D1-depleted GRANTA-519 cells as in A. Results are shown relative to the control (mean $\pm \mathrm{SEM}, n=8$ ), ${ }^{* *} P<0.01,{ }^{* * *} P<0.001$, Student's $t$ test. (F) RNA quantification by pyronin $Y$ staining in control and cyclin D1-depleted GRANTA-519 cells as in B. Left and right panels as in C. Results are shown relative to the control (mean \pm SEM, $n=4$ ), ${ }^{* *} P<0.01,{ }^{* *} P<0.001$, Student's $t$ test. (G) Correlation between cyclin D1 protein levels and pyronin $Y$ staining in $\mathrm{MCL}$ cell lines and cell models. Mean $\pm \mathrm{SEM}, n=4 ; P=4.77 \times 10^{-4}$, mixed-effects models. (H) Pyronin $\mathrm{Y}$ intensity of $7 \mathrm{MM}$ cell lines. The cell lines are represented by squares shaded according cyclin D1 levels. (I and J) Quantification of nuclear EU intensity after 24 hours of cyclin D1 induction in JVM13 cell models $(n=2)(\mathrm{I})$ or following cyclin D1 silencing in the GRANTA-519 cell line $(n=2)(\mathrm{J}) .{ }^{* *} P<0.01,{ }^{* * *} P<0.001$, Student's $t$ test. Holm-Bonferroni correction for multiple comparisons was applied to B, C, E, and $\mathbf{F}$. 
A

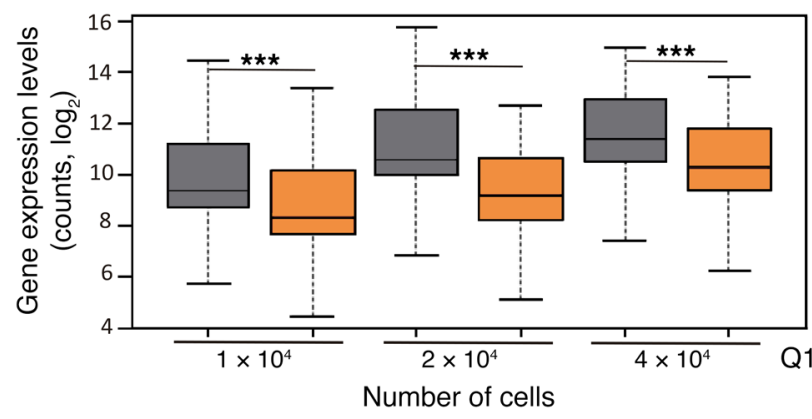

B

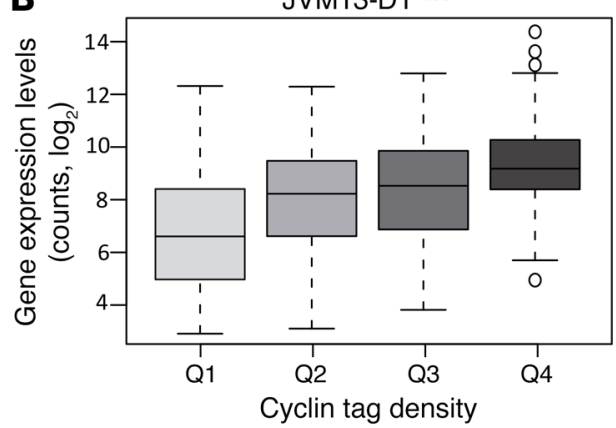

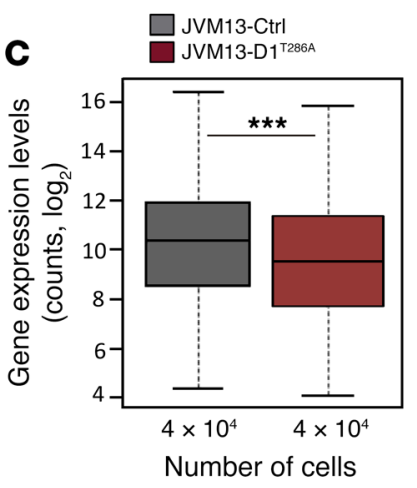

$\square J V M 13-C t r$ JVM13-D1T28e

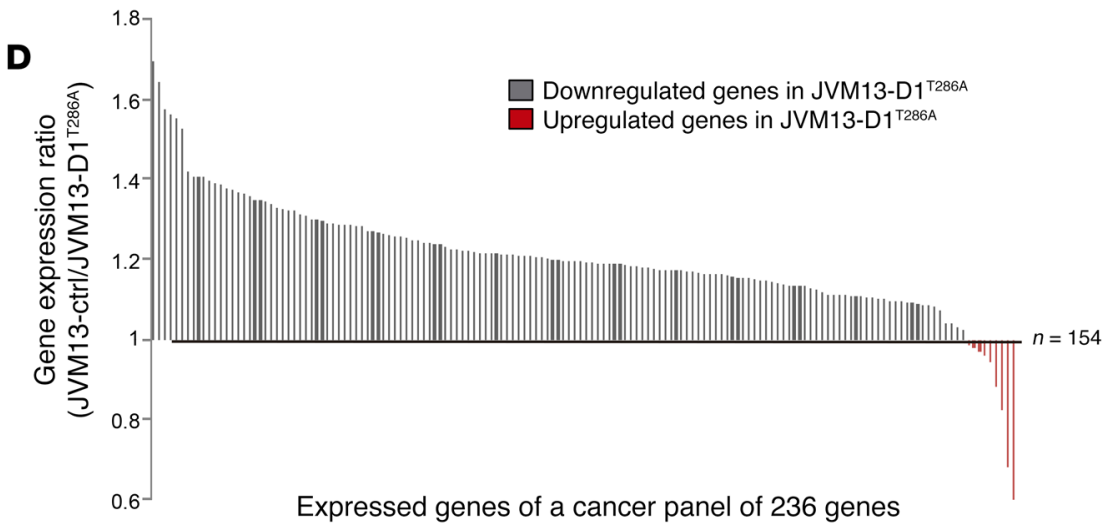

Figure 5. Cyclin D1 overexpression produces a global downmodulation of mRNAs in lymphoid cells. (A) Box plot displaying nCounter-based gene expression data of a 48-gene panel analyzed in JVM13-Ctrl and JVM13-CD1 ${ }^{\text {T286A }}$ cells. Cell extracts from 3 different amounts of cells, counted by cell cytometry, are represented on the $x$ axis. The nCounter counts of expressed transcripts (counts $>30$ ) are shown in $\log _{2}$ scale on the $y$ axis $(n=2)$. ${ }^{* * *} P<2 \times 10^{-16}$, Student's paired $t$ test. (B) Box plot displaying the mean gene expression level in the JVM13-D1 ${ }^{\text {T286A }}$ line of genes expressed in the cancer panel according to cyclin $\mathrm{D} 1$ tag density at promoters ( $-5 \mathrm{~kb}, \mathrm{TSS})$ in JVM13-cD1 ${ }^{\text {T286A }}$ cells distributed in 4 quartiles (Q1-Q4). Cell extracts from $4 \times 10^{4}$ cells were analyzed $(n=2)$. $P=$ $1.7 \times 10^{-6}$, ANOVA. (C) Box plot displaying the mean gene expression level in the JVM13-Ctrl and JVM13-D1 ${ }^{1286 A}$ inducible cell lines of genes expressed in the cancer panel. Cell extracts from $4 \times 10^{4}$ cells were analyzed $(n=2)$. ${ }^{* *} P<2 \times 10^{-16}$, Student's paired $t$ test. (D) Bar plots displaying the gene expression ratio between JVM13-Ctrl and JVM13-D1285A inducible cell lines. Genes, both upregulated (gray) and downregulated (red), are sorted from the highest to the lowest expression ratio over JVM13-Ctrl.

RNA content per cell (Figure 4, E and F). Once again, the cyclin D1 effect was dose related, since the cells with the greatest knockdown efficiency showed the highest increase in RNA amount, and it was cell cycle independent (Supplemental Figure 4D). Then, we investigated whether the different MCL cell lines showed the predicted association between cyclin D1 levels and the amount of total RNA. The analysis identified a highly significant inverse correlation between cyclin D1 protein levels and total RNA amount measured by flow cytometry (Figure $4 \mathrm{G}$ and Supplemental Figure 2E). This association remained significant when exogenous cyclin D1overexpressing and cyclin D1-depleted cells were included $(P<$ $4.77 \times 10^{-4}$; Figure $4 \mathrm{G}$ and Supplemental Figure $\left.4 \mathrm{E}\right)$. We next investigated whether this effect was also present in MM, a lymphoid neoplasm showing cyclin D1 upregulation due to the $\mathrm{t}(11 ; 14)$ translocation or gene amplification in a substantial fraction of the cases. The analysis of MM cell lines with or without the $\mathrm{t}(11 ; 14)$ translocation confirmed in a different cancer cell model the negative correlation between cyclin D1 protein levels and total RNA cell content (Figure $4 \mathrm{H}$ and Supplemental Figure $4 \mathrm{~F}$ ). Finally, RNA quantification in primary MCL cases $(n=11)$ also evidenced a negative correlation between the levels of cyclin D1 and the amount of total RNA, although it did not reach statistical significance $(r=-0.4, P=0.2$; Supplemental Figure $4 \mathrm{G}$ and Supplemental Figure 7C).
In order to test whether this reduction in steady-state RNA levels reflects a decrease in RNA synthesis, we performed nascent RNA-labeling experiments. We measured the incorporation of 5-ethylnyl-uridine (EU) in JVM13, JVM13-D1, and JVM13-D1 ${ }^{\mathrm{T} 286 \mathrm{~A}}$ cells to quantify the rate of nascent transcription at the single-cell level. Interestingly, cyclin D1 overexpression led to a significant reduction in transcription (Figure $4 \mathrm{I}$ and Supplemental Figure 5A). Accordingly, cyclin D1 depletion in the GRANTA-519 cell line revealed a significant increase in RNA synthesis (Figure 4J and Supplemental Figure 5B). Together, these results suggest that cyclin D1 oncogenic overexpression induces a reduction in steady-state RNA levels in a dose-dependent manner by decreasing total transcription.

Cyclin D1 produces a global downmodulation of mRNAs in lymphoid cells. We investigated whether the reduced transcription promoted by cyclin D1 overexpression also determines a global mRNA downregulation. The decrease in the quantity of total RNA per cell following cyclin D1 overexpression compromises the use of genome-wide approaches such as microarrays or RNA-Seq to explore the presence of a global mRNA downmodulation, since these strategies usually compare the same amount of RNA between conditions (30). To overcome this limitation, we took advantage of NanoString's nCounter technology, which is able to perform a 

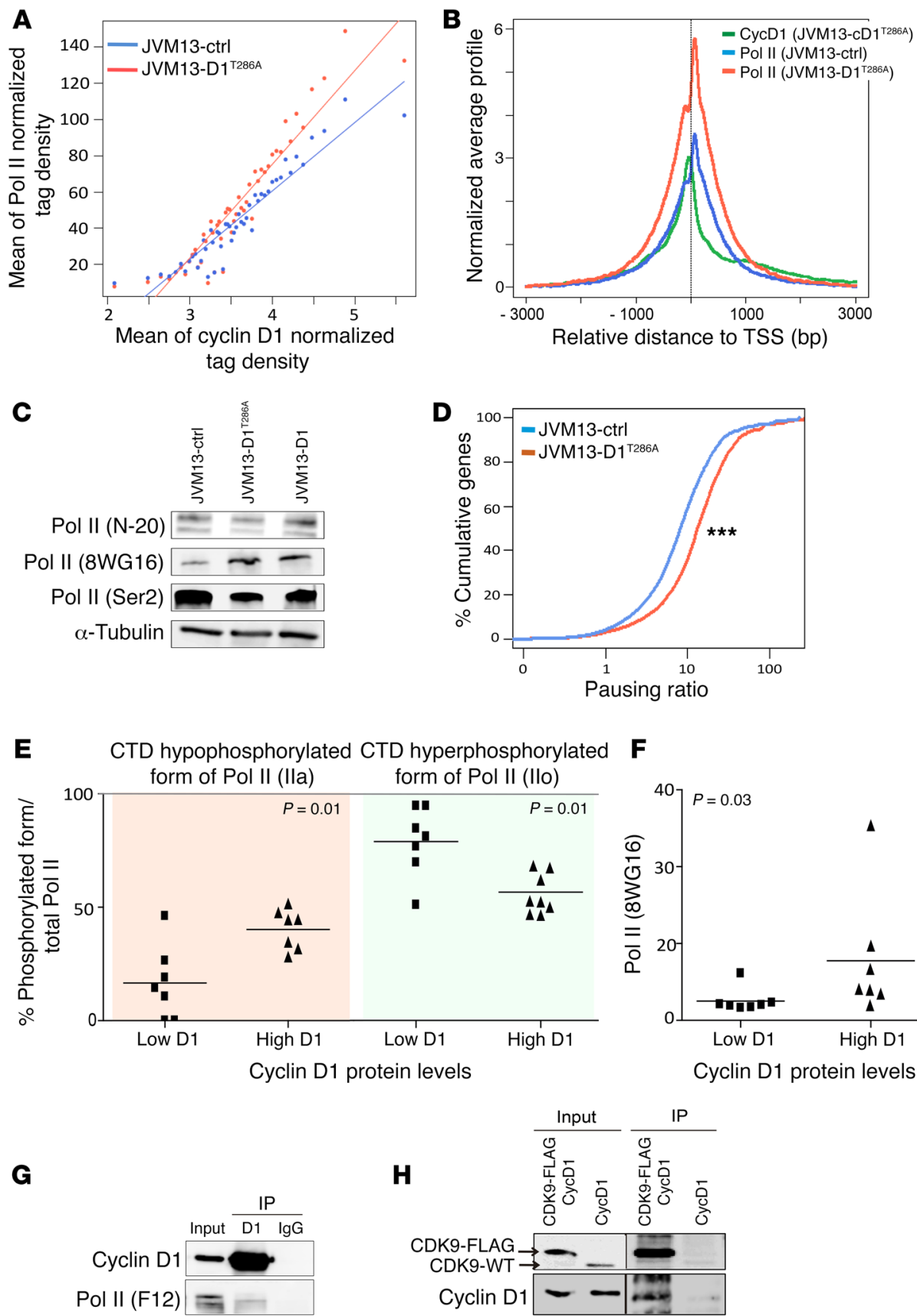

Figure 6. Cyclin D1 colocalizes with RNA Pol II and promotes an increase in the Pol II pausing index. (A) Correlation between normalized cyclin D1 ChIP-Seq tag density in JVM13-cD1 ${ }^{1286 A}$ and Pol II ChIP-Seq tag density at promoters in JVM13-ctrl and JVM13-D1 ${ }^{1286 A}$ cells. Promoters were sorted into 50 equalsized groups based on ChIP-seq tag densities of cyclin D1. The $x$ axis represents mean cyclin D1 normalized tags of the promoters in JVM13-CD1 ${ }^{\text {T286A }}$ cell lines. The $y$ axis represents Pol Il tag density in both cell lines. The linear regression line between cyclin D1 and Pol II presence in promoters is shown. (B) Average signal profiling of Pol II occupancy around the TSS ( $\pm 3 \mathrm{~kb}$ ) in JVM13-ctrl and JVM13-D1 ${ }^{\text {T286A }}$ inducible cell lines. The cyclin D1-binding profile in JVM13-cD1 ${ }^{\text {T286A }}$ cells is also shown. (C) Western blot showing different phosphorylated forms of Pol II in JVM13-ctrl, JVM13-D1 ${ }^{\text {T286A }}$, and JVM13-D1 inducible cell lines. Gels were run in duplicate for the study of the phosphorylation forms. A representative Western blot $(n=3)$ for each antibody is presented. $\alpha$-Tubulin of only one of the gels run in duplicate is shown as loading control. (D) Plot representing the pausing index. Lines illustrate rightward shift of pausing ratio at all genes with cyclin $\mathrm{D} 1$ in their promoter $(-5$ $\mathrm{kb}, \mathrm{TSS})$ after cyclin D1 induction in JVM13-ctrl and JVM13-D1 $1^{\text {T286A }}$ cells. ${ }^{* * *} P<2 \times 10^{-16}$,

Kolmogorov-Smirnov test. (E) Proportion of Pol II (Ilo) and Pol (Ila) forms in primary MCL cases. $P=0.01$, nonparametric Mann-Whitney $U$ test. (F) Pol II (8WG16) antibody signal in primary MCL cases. $P=0.03$, nonparametric Mann-Whitney $U$ test. (G) Coimmunoprecipitation experiment in Z-138 cells using antibodies against cyclin D1 and control lgG. Immunoprecipitated proteins were analyzed by Western blot by blotting with cyclin D1 and Pol II antibody. Input at $1 \%$ was loaded as a control. (H) Coimmunoprecipitation experiment in HEK-293T-CDK9-FLAG-D1 ${ }^{\text {T286A }}$ cells with anti-FLAG resins. Immunoprecipitated proteins were analyzed by Western blot by blotting with CDK9 and cyclin D1 antibodies. HEK-293T-D1 ${ }^{\text {T286A }}$ immunoprecipitation was used as negative control. direct digital quantification of cell extracts. We hybridized a commercial predefined panel containing 48 genes derived from the MicroArray Quality Control Study (MAQC) (31) with cell extracts from 3 different amounts of cells corresponding to the cyclin D1 overexpression model (JVM13 and JVM13-cD1 ${ }^{\mathrm{T} 286 \mathrm{~A}}$ ). The high correlation observed between mRNA counts and the number of cells confirmed the suitability of this approach to test the global effect of cyclin D1 (JVM13-control, $r=0.969 \pm 0.016$; JVM13-cD1 ${ }^{\mathrm{T} 286 \mathrm{~A}}, r=$ $0.987 \pm 0.007$; Figure 5A). In accordance with the total RNA quantification data, the cells overexpressing cyclin D1 showed lower gene expression levels than control cells at the 3 amounts of cells $\left(P<2.2 \times 10^{-16}\right.$; Figure 5A). Interestingly, all 36 genes expressed in control cells were downregulated in cyclin D1-overexpressing cells. To further confirm the global impact of cyclin D1 on mRNA levels, we analyzed the expression of a commercial predesigned panel of 236 cancer-related genes in the JVM13-D1 ${ }^{\text {T286A }}$ cell line following cyclin D1 induction. Control cells expressed 154 of the 236 genes. Observing results consistent with the RNA-Seq results, we found an association between the cyclin D1 tag density and the gene expression levels in JVM13-D1 ${ }^{\mathrm{T} 286 \mathrm{~A}}$ cells (Figure $5 \mathrm{~B}, P$ $\left.=1.7 \times 10^{-6}\right)$. Remarkably, the average expression of these genes was significantly lower in JVM13-D1 ${ }^{\text {T286A }}$ cells (Figure 5C). 94\% $(n=145)$ of the genes expressed in control cells were expressed at lower mRNA levels in JVM13-D1 ${ }^{\mathrm{T} 286 \mathrm{~A}}$ cells (Figure 5D). To explore whether this mRNA downmodulation would include tumor suppressor genes (TSGs), we compiled a set of 583 genes defined as TSGs (32). We found that 30 of 33 (91\%) expressed TSGs were downregulated in JVM13-D1 ${ }^{\mathrm{T} 286 \mathrm{~A}}$ cells. Interestingly, the TSGs 

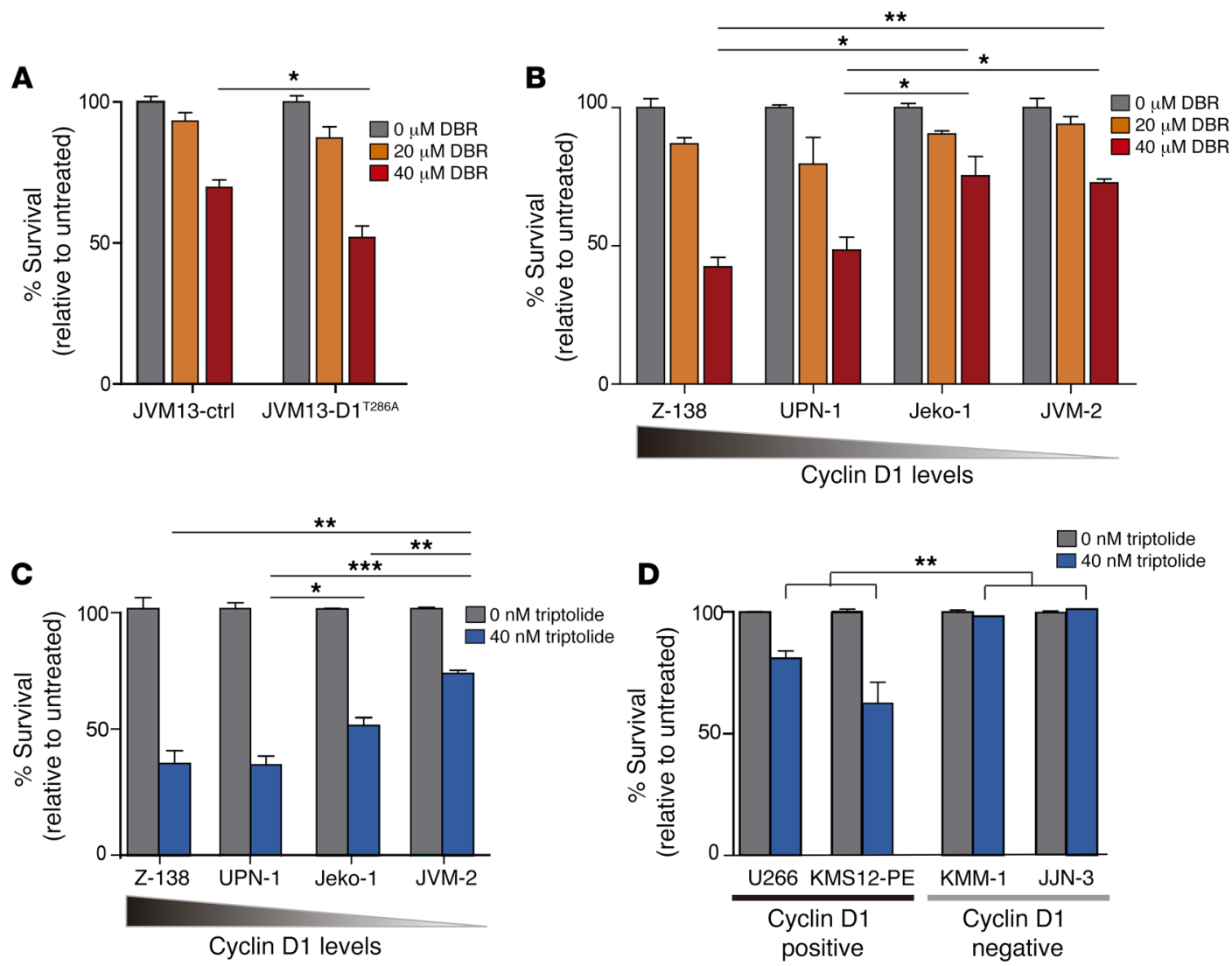

$\mathbf{E}$

DBR

$\mathbf{F}$

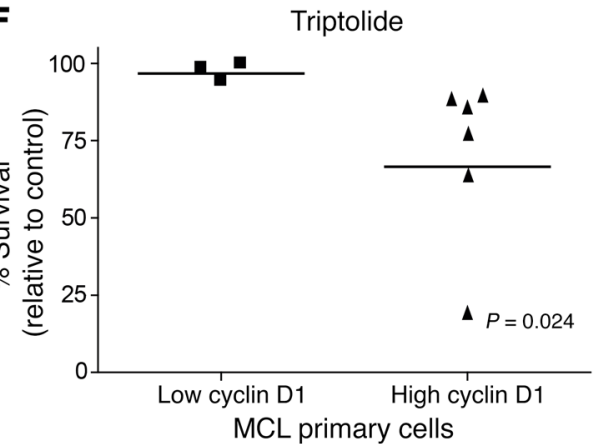

Figure 7. Cyclin D1 overexpression renders tumor cells sensitive to CDK9 inhibitors. (A) Cell survival of cyclin D1 inducible model after treatment with a CDK9 inhibitor (DBR) at increasing concentrations. JVM13-Ctrl and JVM13-D1 ${ }^{\text {T286A }}$ cells, after 24 hours of doxycylcine induction, were treated during 48 hours with DBR. Results are shown as mean \pm SEM with respect to untreated cells (mean $\pm S E M, n=3$ ). ${ }^{*} P<0.05$, Student's $t$ test. (B) MCL cell lines were treated during 72 hours with DBR. Results are shown with respect to untreated cells (mean \pm SEM, $n=4$ ). ${ }^{*} P<0.05,{ }^{* *} P<0.01,{ }^{* *} P<0.001$, Student's $t$ test. (C and D) Cell survival of MCL cell lines (C) and MM cell lines (D) after treatment with triptolide at $40 \mathrm{nM}$. Exponentially growing cell lines were treated, and cell survival was measured at 48 hours. Results are shown with respect to untreated (mean \pm SEM $n=4$ ). ${ }^{*} P<0.05$, ${ }^{* *} P<0.01$, ${ }^{* * *} P<0.001$, Student's $t$ test (C) or mixed-effects models (D). (E and F) Cell survival of 9 primary MCL cases after treatment with $60 \mu \mathrm{M}$ DBR (E) or $200 \mathrm{nM}$ triptolide (F). Cell survival was measured at 72 hours after treatment. Survival was calculated with respect to untreated controls, and the means of duplicate experiments for each group are represented. Nonparametric Mann-Whitney $U$ test was applied. Holm-Bonferroni correction for multiple comparisons was applied to A-C.

analyzed in the cancer panel more frequently showed cyclin D1 peaks at their promoters than the rest of the genes $(81 \%$ vs. $61 \%, P$ $=0.038$, Supplemental Figure 6A). Then we explored the binding of cyclin D1 to the promoters of the 583 TSGs in the MCL cell lines. We observed that a significantly greater fraction of TSGs showed cyclin D1 peaks in MCL cell lines (48\% vs. $36 \%, P<3.3 \times 10^{-9}$, Supplemental Figure 6B). Concordantly, the analysis of TSGs in primary MCL cases evidenced that this set of genes tends to show high expression levels in MCL cases (Supplemental Figure 6C).
Together, these results strongly suggest that the binding of cyclin D1 to gene promoters leads to a global transcription downmodulation of expressed mRNAs also involving TSGs.

Cyclin D1 physically interacts with the transcription machinery and increases promoter-proximal Pol II pausing. To explore whether the cyclin D1-dependent transcriptome downmodulation was associated with changes in the RNA Pol II chromatin binding profile, we performed Pol II ChIP-Seq experiments in control (JVM13) and cyclin D1-overexpressing cells (JVM13-D1 ${ }^{\mathrm{T} 286 \mathrm{~A}}$ ) (Supplemen- 


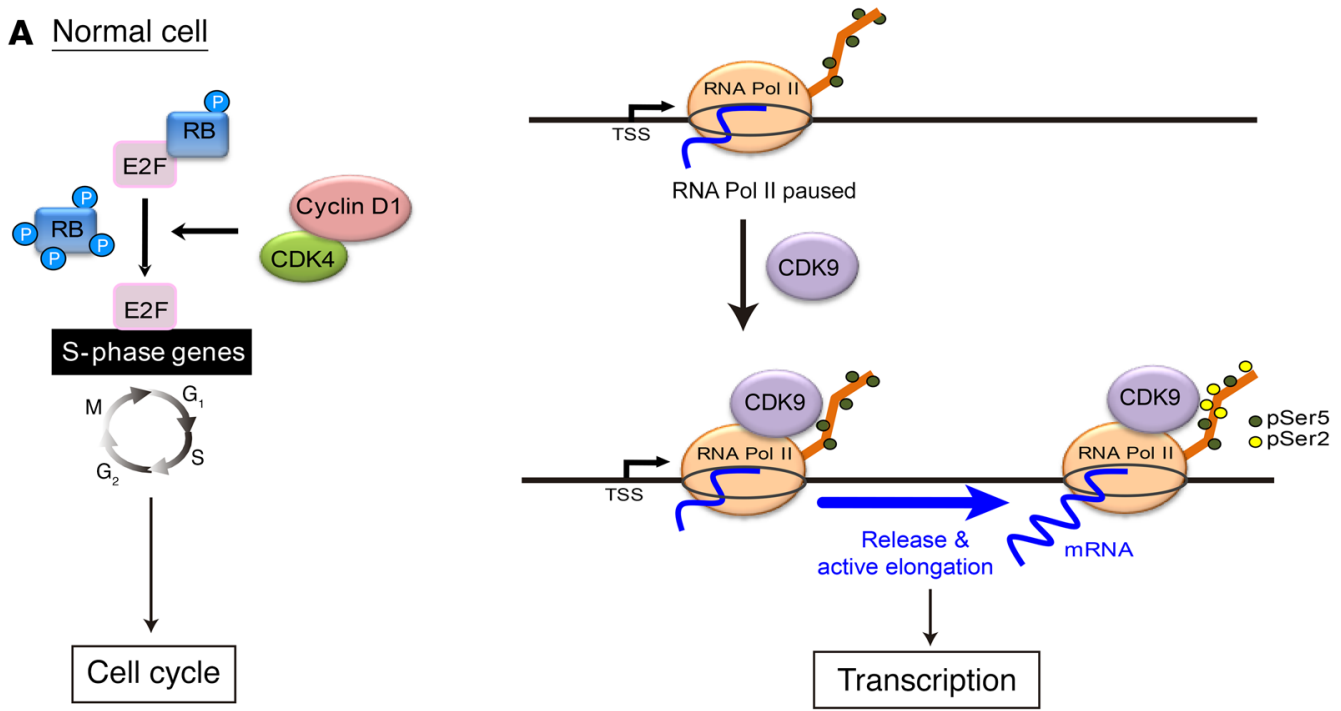

Figure 8. Proposed model for cyclin D1-dependent global transcriptional downregulation, a side effect of its oncogenic overexpression. (A) Cyclin D1 canonical cell cycle role in normal cells. (B) Overexpression of cyclin D1, in addition to cell cycle induction, may interact with CDK9 and interfere with the normal release of paused $\mathrm{Pol}$ II, compromising active elongation. This would lead to a global transcription downmodulation, including of TSGs. The dotted lines indicate other potential oncogenic effects, such increased genomic instability due to augmented conflicts between transcription and DNA replication machinery.

B Cancer cell overexpressing cyclin D1

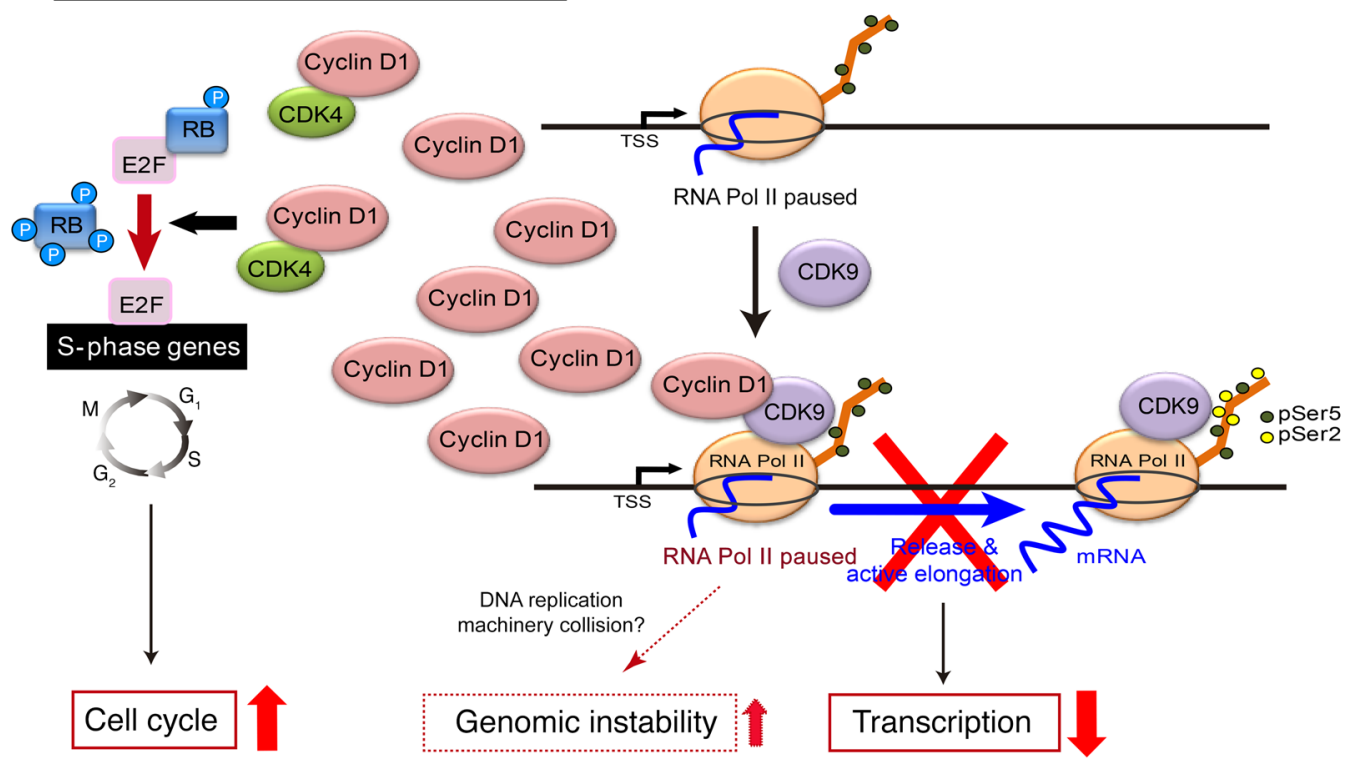

tal Figure 7A). Observing results concordant with the association between cyclin D1 chromatin binding and transcriptional levels, we found a strong correlation between the tag density of cyclin D1 and Pol II together with a colocalization of both binding profiles around the TSS (Figure 6, A and B). Interestingly, JVM13-D1 ${ }^{\mathrm{T} 286 \mathrm{~A}}$ cells showed higher Pol II loading at promoters, and this difference was more evident in highly expressed genes (Figure 6B and Supplemental Figure 7B). These results suggest that the observed transcriptome downmodulation was not due to reduced Pol II recruitment to promoters, but was related to increased promoterproximal Pol II pausing. Consistent with this scenario, cyclin D1overexpressing cells displayed levels of Pol II protein similar to those in control cells (Figure 6C; Pol II [N-20]), indicating that the increased promoter occupancy by Pol II was not due to the presence of higher Pol II levels. Interestingly, the phosphorylation status of the C-terminal domain (CTD) of Pol II was different in cyclin D1-expressing cells as compared with the control cells. Cyclin D1overexpressing cells showed lower levels of Ser2 phosphorylation, a CTD modification associated with active elongation (Figure 6C). Concordantly, the 8WG16 antibody, which binds to unphosphorylated CTD and more weakly to Ser5-phosphorylated CTD, showed higher signal intensity in cyclin D1-overexpressing cells (Figure 6C and ref. 33). These Pol II forms are associated with paused polymerase or early elongation complexes. This Pol II phosphorylation pattern supports the view that cyclin D1 overexpression increases promoter-proximal Pol II pausing. To confirm whether the Pol II pause release was affected by overexpression of cyclin D1, we established the pausing index by determining the ratio between Pol II occupancy at promoters and gene body regions (34). This analysis showed that cyclin D1 overexpression led to an augmented Pol II pausing index (Figure 6D). Together, these results strongly support that the reduction of total nascent transcription following cyclin D1 overexpression observed in lymphoid cells was due to increased promoter-proximal Pol II pausing.

To investigate whether the phosphorylation pattern of Pol II could be dysregulated in primary MCL cases, we analyzed the 
expression of Pol II in a series of cases $(n=14)$. All samples showed expression of the CDT hyperphosphorylated form of Pol II (IIo), whereas expression of the hypophosphorylated CTD form (IIa) was heterogeneous among primary MCL cases (Supplemental Figure 7C). We established a ratio between the levels of the IIa and IIo forms and the amount of total Pol II. When we classified the primary MCL cases in 2 groups based on their low or high cyclin D1 protein levels (Supplemental Figure 7C), we observed that the proportion of the hyperphosphorylated Pol II (IIo) form was significantly greater in MCL cases with low cyclin D1 levels (Figure $6 \mathrm{E})$, whereas the MCL cases with high cyclin D1 showed a significantly greater proportion of the hypophosphorylated form (IIa) (Figure 6E). This association between the hypophosphorylated form of the Pol II and the levels of cyclin D1 was confirmed using the 8 WG16 antibody (Figure 6F and Supplemental Figure 7C). Overall, these results suggest that the overexpression of cyclin D1 may impact the transcription process by dysregulating RNA Pol II CDT phosphorylation.

We next explored whether the transcription dysregulation promoted by cyclin D1 overexpression could be mediated by an interaction of the cyclin with the transcription machinery. Cyclin D1 coimmunoprecipitation analysis in MCL cell lines showed that indeed cyclin D1 interacts with Pol II (Figure 6G and Supplemental Figure 8A). Furthermore, the association found between the amount of cyclin D1 and the hypophosphorylation pattern compatible with paused Pol II led us to investigate whether this phenotype could be mediated by an off-target effect of the overexpressed cyclin D1 on the cyclin-dependent kinase CDK9. This CDK is the kinase subunit of positive transcription elongation factor $\mathrm{b}(\mathrm{P}-\mathrm{TEFb})$ required for release of paused Pol II and responsible of Ser2 phosphorylation during active elongation (35). In order to test whether cyclin D1 interacts with CDK9, we transduced HEK-293T cells with cyclin D1 (D1 ${ }^{\mathrm{T} 286 \mathrm{~A}}$ ) and CDK9 (CDK9-Flag) and performed coimmunoprecipitation experiments. Interestingly, we observed an interaction between CDK9 and cyclin D1 proteins (Figure 6H). Moreover, endogenous coimmunoprecipitation experiments in MCL cell lines confirmed this cyclin D1-CDK9 interaction (Supplemental Figure $8, \mathrm{~B}$ and $\mathrm{C}$ ). These results suggest that cyclin D1 overexpression may dysregulate the release of paused Pol II through its binding to CDK9. Together, these results indicate that cyclin D1 overexpression determines a global transcriptome downmodulation by interacting with the transcription machinery and interfering with the release of promoter-proximal paused Pol II.

Cyclin D1 overexpression sensitizes cells to transcription inhibitors. The global transcriptional downmodulation caused by cyclin D1 overexpression led us to hypothesize that cyclin D1 overexpression could render cells more sensitive to drugs targeting the transcription machinery. To explore whether cyclin D1 overexpression sensitizes cells to transcription inhibitors, we treated control (JVM13) and cyclin D1-overexpressing (JVM13-D1 ${ }^{\mathrm{T} 286 \mathrm{~A}}$ ) cells with 5,6-dichloro-1- $\beta$-ribofuranosyl benzimidazole (DRB), a transcription inhibitor that causes premature chain termination, with CDK9 being its major target. We used DRB at 2 different concentrations $(20 \mu \mathrm{M}$ and $40 \mu \mathrm{M})$ below the levels reported to fully inhibit transcription $(100 \mu \mathrm{M})(36)$. This experiment demonstrated significantly increased sensitivity to DRB in cyclin D1-overexpressing cells compared with control cells (Figure 7A). To explore whether the sensitivity to transcription inhibitors was related to the cyclin D1 levels, we analyzed the response of MCL cell lines to DRB. The MCL cell lines showed a significant increase in apoptosis following DRB treatment (Figure 7B). Interestingly, the MCL cell lines with higher amounts of cyclin D1 and lower RNA content per cell showed greater sensitivity to DRB (Z-138 and UPN-1).

To further investigate the synthetic lethality interaction between cyclin D1 overexpression and transcription inhibition, we treated MCL and MM cell lines with triptolide, a transcription inhibitor that has been used for cancer treatment, with promising results (37). This inhibitor promotes the degradation of RPB1, the largest subunit of RNA Pol II (38). Again, UPN1 and Z-138 showed the greatest sensitivity to triptolide (Figure 7C). Interestingly, the cyclin D1-positive MM cell lines that showed a smaller amount of total RNA also displayed a significantly greater sensitivity to triptolide than cyclin D1-negative MM cell lines (Figure 7D). These results suggest that high cyclin D1 expression leads to increased sensitivity to transcription inhibitors.

To test whether this synthetic lethality interaction could be observed in primary MCL cases, we treated cells from MCL patients with DRB and triptolide. We observed that primary MCL cells with higher levels of cyclin D1 were more sensitive to DRB and triptolide than MCL cells with smaller amounts of cyclin D1 (Figure 7, E and F). Overall, these results suggest the existence of a potential synthetic lethality interaction between the overexpression of cyclin D1, responsible for a global transcription downmodulation, and transcription inhibitors.

\section{Discussion}

We investigated whether the oncogenic overexpression of cyclin D1 is responsible for a transcriptional dysregulation in lymphoid tumor cells. As a model, we used MCL cells characterized by the constitutive overexpression of cyclin D1 due to the $\mathrm{t}(11 ; 14)$ translocation. We found that endogenous cyclin D1 showed widespread binding to promoter regions of active genes, and its overexpression was responsible for a global transcriptional downmodulation in these malignant B cells. This dysregulation seems to be mediated by the interference of the overexpressed cyclin D1 with the release of promoter-proximal paused Pol II. The interaction of overexpressed cyclin D1 with the transcription machinery and the consequent global RNA downmodulation rendered the tumor cells more sensitive to transcription inhibitors.

Our genomic analysis of cyclin D1-chromatin interaction revealed a high and somewhat unexpected number of cyclin D1 binding sites that positively correlated with cyclin D1 protein levels and preferentially occurred at promoters close to the TSS. Analysis of the relationship between the cyclin D1 promoter occupancy and chromatin states together with the transcriptional output demonstrated that cyclin D1 binds globally to active promoters and these interactions are proportional to the mRNA abundance of targeted genes. Our results are in agreement with the observation that exogenous cyclin D1 targets promoters of genes highly expressed during retina development in cyclin D1-knockin mouse embryos (39). The density of cyclin D1 peaks in MCL cells was higher than that reported recently for exogenous cyclin D1 in mouse cancer cell lines and retinal tissue (39-41). This difference could be explained by the extremely high cyclin D1 protein levels 
present in MCL cell lines. Furthermore, we observed that exogenous cyclin D1 was recruited to promoters that are preconfigured in an active state in the absence of cyclin D1, and the recruitment seems to be proportional to the expression levels of the targeted genes. This chromatin behavior was strikingly similar to that reported for MYC in human tumor cells and mouse primary lymphocytes $(26-28,42)$. Recent studies have associated the MYC chromatin-binding profile with its capacity to globally enhance transcription at all active loci, giving rise to the model of MYC as a global transcriptional amplifier $(26,27)$.

Surprisingly, despite the substantial similarities between the chromatin-binding behavior of cyclin D1 and MYC, the overexpression of cyclin D1 in lymphoid cells was responsible for a significant reduction in cell RNA content that was proportional to cyclin D1 protein levels, an effect that was cell cycle independent and was related to a reduced transcription rate. The analysis of MCL and MM cell lines and primary MCL cases confirmed the negative correlation between cyclin D1 protein levels and total RNA cell content. Moreover, the digital quantification of 2 independent gene sets showed that cyclin D1 overexpression also determined a downregulation of most expressed mRNA transcripts. These results suggest that the widely spread binding of cyclin D1 to promoters decreases the nascent transcription of bound genes, producing a significant reduction in cellular mRNA content. Thus, cyclin D1 seems to act as global transcriptome regulator, decreasing the overall gene transcription program of the cells. As far as we know, this is the first time that a global transcription downregulation effect has been reported for a protein outside the basal transcription machinery.

The phosphorylation state of the CTD of Pol II is critical in determining its activity during the transcription process $(43,44)$. Initially, Pol II is recruited into the pre-initiation complex with a hypophosphorylated CTD (IIa), and later, during initiation, the CTD is phosphorylated on Ser5 mainly by CDK7 and on Ser2 during active elongation by CDK9, the kinase component of P-TEFb, resulting in Pol II hyperphosphorylation (IIo). We observed that the overexpression of cyclin D1 determined a shift in the phosphorylation pattern of the Pol II CTD from a productive elongation to a paused polymerase pattern (35). Moreover, primary MCL cases with higher levels of cyclin D1 showed an increased proportion of hypophosphorylated forms of Pol II (IIa). These phosphorylation patterns were consistent with the observed accumulation in promoterproximal regions of paused Pol II following cyclin D1 overexpression. Conversely, the global MYC-dependent transcription amplification was related to the capacity of MYC to increase Pol II processivity at all transcribed genes $(26,27,45,46)$. In light of these results, we postulated that the phosphorylation pattern shift and the global transcriptional downmodulation we observed could be mediated by the physical interaction of cyclin D1 with the transcription machinery. This scenario is supported by the coimmunoprecipitation of cyclin D1 with RNA Pol II and CDK9 observed in MCL cell lines and in HEK-293T cells. Previous studies have reported the interaction of cyclin D1 with CDKs besides the canonical CDK4/6 in different cell models, including CDK1, CDK2, CDK3, CDK5, and CDK11 (39, 47-49). Furthermore, the binding of cyclin D1 to noncanonical CDK partners has been described to inhibit the targeted CDK (50). In a similar way, the binding of cyclin D1 could sequester CDK9 in inactive complexes, compromising the CDK9-dependent Ser2 phosphorylation required for active elongation. This would give rise to the accumulation of paused RNA Pol II and the global transcriptional downmodulation we identified (Figure 8). Further analyses should clarify in more detail how cyclin D1 interferes with the activation of the transcription machinery, whether other CDK9 functions are compromised, and whether off-target effects may be a common phenomenon following the pathogenic overexpression of other cyclins.

The increased sensitivity to a CDK9 inhibitor displayed by lymphoid cells following cyclin D1 overexpression together with the significantly greater sensitivity to transcription inhibitors observed in MCL and MM cell lines and in primary MCL cases with higher cyclin D1 levels suggests the existence of a synthetic lethality between cyclin D1 overexpression and transcription inhibition. This lethal interaction opens opportunities for new treatment strategies in cyclin D1-overexpressing tumors. In this sense, a recent study has reported that SNS-032, a CDK7/9 inhibitor, induces cytotoxicity in MCL cells (51). Interestingly, consistent with our results, the SP-53 MCL cell line, which displays very low levels of cyclin D1 (52), was the cell line least sensitive to SNS-032 (51).

The identification of a cyclin D1-dependent global transcriptional downmodulation may seem at first glance inconsistent with a potential oncogenic effect of cyclin D1 in MCL, and raises the question about its potential significance. We may hypothesize that this transcriptional downmodulation could be a mechanism that participates in the transcriptional shutdown during the $S$ phase following the $G_{1} / S$ transcriptional wave or during M-phase in a normal cell cycle $(53,54)$. This dysregulation also could correspond to a nonphysiological collateral effect produced by the very high levels of cyclin D1. Further studies should clarify whether this downmodulation responds to a physiological role of cyclin D1.

The hypothesis that the transcriptional downregulation mediated by high levels of cyclin D1 plays a potential oncogenic role in MCL is suggested by the fact that MCL cells accumulate other genetic alterations, in addition to the $\mathrm{t}(11 ; 14)$ (q13; q32) translocation, that lead to increased cyclin D1 levels, emphasizing that cyclin D1-dependent phenotypes might be important in MCL pathogenesis. Interestingly, the presence of more stable cyclin D1 transcripts and higher protein levels correlates with increased aggressiveness of the tumors (22). Although this aspect requires further study, we may speculate that a reduction of the transcriptome program of a cell could have an oncogenic impact by decreasing the levels of active TSGs, including cell cycle checkpoint genes, which are frequently inactivated or downregulated during oncogenesis (Figure 8). Also, we may hypothesize that the accumulation of paused Pol II due to cyclin D1 overexpression might facilitate the generation of genomic instability by increasing the probability of conflicts between DNA replication and transcription machineries. The collision between these machineries may cause an increase in DNA breaks as a consequence of replication fork stalling and collapse leading to recombination and chromosome rearrangements (Figure 8 and ref. 55). In this sense, it has been recently shown that artificially stabilized transcription initiation complexes can impede replication fork progression (56). Further studies should clarify the potential conflicts between promoter-proximal paused Pol II mediated by cyclin D1 overexpression and DNA replication forks. 
In conclusion, we have identified that oncogenic cyclin D1 overexpression produces a global transcriptome downmodulation. This dysregulation might, at least in part, be mediated by the interaction of cyclin D1 with transcription machinery that would compromise the release of promoter-proximal paused Pol II. This global transcriptional downmodulation induced by cyclin D1 overexpression seems to generate a synthetic lethality interaction that could be exploited therapeutically.

\section{Methods}

Further information can be found in Supplemental Methods, available online with this article.

Cell lines and primary MCL cells. For this study, we used 5 wellcharacterized MCL cell lines (Z-138, GRANTA-519, JeKo-1, UPN-1, and JVM-2) (24), 7 MM cell lines (JJN-3, RPMI 8226, NCI-H929, U266, KMS-12-PE, KMM-1, and ARP-1; purchased from the German Collection of Microorganisms and Cell Cultures and ATCC), a lymphoblastoid leukemic cell line (JVM-13; ATCC CRL3003), and HEK293T cells (ATCC CRL-3216). Cells were cultured at $37^{\circ} \mathrm{C}$ and $5 \%$ $\mathrm{CO}_{2}$ in RPMI (Gibco), except for GRANTA-519 and HEK-293T cells in DMEM (Gibco). Media was supplemented with 10\% FBS, $500 \mu \mathrm{g} / \mathrm{ml}$ streptomycin, and $500 \mathrm{U} / \mathrm{ml}$ penicillin (Gibco).

Primary MCL samples ( $>80 \%$ tumor cells) were obtained from peripheral blood samples of $14 \mathrm{MCL}$ patients diagnosed according to WHO criteria. Tumor cells were isolated by centrifugation on a FicollHypaque (GE Healthcare) gradient, cryopreserved, and maintained in the Hematopathology collection registered at the Biobank of Hospital Clínic-IDIBAPS (R121004-094).

Plasmids and infection procedures. Plasmids for constitutive infection used the backbone pBABE-puro retroviral vector. pBABE-puro was a gift from Hartmut Land, Jay Morgenstern, and Bob Weinberg (Addgene, plasmid 1764) (57). pBABE-puro-cyclinD1 (tagged with the 27-base pair sequence HA) was a gift from William Hahn (Addgene, plasmid 9050). pBABE-Flag-Cdk9-IRES-eGFP was a gift from Andrew Rice (Addgene plasmid 28096) (58). Mutation T286A in the cyclin D1 gene was inserted under the manufacturer's guidelines using a QuikChange II Site-Directed Mutagenesis Kit (Agilent). All constructs were validated by sequencing. For cyclin D1 knockdown, we used MISSION pLKO.1-puro plasmids (Sigma-Aldrich) (Supplemental Table 4).

Constitutive overexpression models were achieved by spininfection of JVM-13 or HEK-293T exponentially growing cells $(1,350 \mathrm{~g}$, 90 minutes, $27^{\circ} \mathrm{C}$ ) in the presence of Polybrene (Sigma-Aldrich) and supernatants containing viral particles produced in HEK-293T cells. HEK-293T CDK9-FLAG-GFP-infected cells were sorted by cell cytometry (BD LSRFortessa, BD Biosciences). Cyclin D1 constitutive models were selected after 1 week of puromycin treatment at $0.4 \mu \mathrm{g} /$ $\mathrm{ml}$ (Sigma-Aldrich). The inducible cell lines JVM-2 and JVM-13 were developed under the manufacturer's guidelines using the plasmids of the Retro-X Tet-On Advanced Inducible Expression kit (Clontech Laboratories). Silencing of cyclin D1 in exponentially growing GRANTA-519 cells was performed by lentiviral spin infection $(1,000$ $g, 90$ minutes, $32^{\circ} \mathrm{C}$ ) in the presence of Polybrene, followed by an additional 3 hours of incubation; the protocol was repeated for 2 consecutive days. Cells were selected with $0.5 \mu \mathrm{g} / \mathrm{ml}$ puromycin (SigmaAldrich) 24 hours after infection.

In vitro transcription inhibition experiments. Exponentially growing MCL cell lines and cyclin D1 inducible cells incubated for 24 hours in the presence of doxycycline $(0.1 \mu \mathrm{g} / \mathrm{ml})$ were treated with DRB (SigmaAldrich) or triptolide (Selleck Chemicals) at the indicated final concentrations. The annexin-positive fraction was determined by cell cytometry 48 hours after DRB treatment or 72 hours after triptolide treatment using an Annexin V Apoptosis Detection Kit FITC (eBioscience) according to the manufacturer's guidelines. Nine MCL primary samples were treated for 72 hours with DRB or triptolide at the indicated concentrations, and apoptosis was analyzed in the CD19-positive fraction (CD19-PE, Becton Dikinson) using the Annexin V Apoptosis Detection Kit FITC.

ChIP. For cyclin D1, crosslinked chromatin was fragmented with a Covaris S220 instrument using the truChIP HighCell Chromatin Shearing Kit with Non-ionic Shearing Buffer. Immunoprecipitation was performed with anti-cyclin D1 antibody or control IgG. Chromatin was de-crosslinked and purified with Agencourt AMPure beads (Beckman Coulter) according to the manufacturer's guidelines. 1\% of sheared DNA was used as input control for qPCR validation and for analyzing sonication efficiency. Equal volumes of eluted chipped DNA were amplified using SYBR Green PCR Master Mix (Applied Biosystems) under the manufacturer's guidelines. Specific primers were designed for the peaks obtained in the ChIP-Seq analysis (Supplemental Table 3). Primers for negative regions were also designed to test the specificity of the enrichment. For Pol II, crosslinked chromatin was sonicated with a Bioruptor (Diagenode). Shared chromatin was immunoprecipitated using anti-Pol II antibody, or control IgG chromatin was decrosslinked and purified using the phenol-chloroform method. $1 \%$ of sheared DNA was used as input control for qPCR validation and for analyzing sonication efficiency.

ChIP-Seq and RNA-Seq. Library preparation for ChIP-Seq was performed using the NEBNext Ultra DNA Library Prep Kit (New England BioLabs). Briefly, the immunoprecipitated DNA (10 ng) was endrepaired, and A-nucleotide overhangs were then added, followed by adapter ligation, PCR enrichment (15 cycles), and purification with AMPure beads (ratio, 1.25) (Beckman Coulter). The purified DNA library products were evaluated and quantified using Bioanalyzer (Agilent, High Sensitivity DNA Kit) and the KAPA Library Quantification Kit (Kapa Biosystems), respectively. Sequencing was performed on the Illumina MiSeq instrument using the 50-cycles V2 kit. For RNA-Seq, total RNA was isolated using TRIzol (Zymo Research). Preparation of RNA-Seq libraries was carried out using the TruSeq RNA Sample Preparation Kit (Illumina) according to the manufacturer's standard protocol. The libraries were 75-bp paired-end sequenced at approximately 80 million reads per library with Illumina technology. RNASeq data were deposited in the NCBI's Gene Expression Omnibus database (GEO GSE118301)

RNA extraction and quantification by flow cytometry. Cells were counted in duplicate from cell cultures using FACS (Attune NxT, Thermo Fisher Scientific). Total RNA was extracted from 1 million cells using an RNeasy Mini Kit (QIAGEN) under the manufacturer's guidelines. RNA extracts were quantified by Nanodrop ND-1000 (Thermo Fisher Scientific). Cell pellets of 1 million cells were fixed in $0.5 \%$ formaldehyde for 15 minutes on ice. Cells were pelleted, washed with cold PBS, and resuspended in $300 \mu \mathrm{l}$ cold PBS. Then $700 \mu \mathrm{l}$ of $100 \%$ cold ethanol was added dropwise during vortexing. The cells were incubated overnight at $-20^{\circ} \mathrm{C}$. Cell pellets were washed once with PBS at room temperature and resuspended in $250 \mathrm{ml}$ HBSS medium with calcium and magnesium. This suspension was incubated with a 
final concentration of $2 \mathrm{ng} / \mathrm{ml}$ Hoechst 33342 (Sigma-Aldrich) and 4 $\mathrm{ng} / \mathrm{ml}$ Pyronin Y (Sigma-Aldrich) for 20 minutes at room temperature. Cells were analyzed using BD LSRFortessa SORP (BD Biosciences). Hoechst 33342 was excited by a 355-nm laser and emitted at $450 \mathrm{~nm}$. Pyronin Y was excited by a $488-\mathrm{nm}$ laser, emitted at $560 \mathrm{~nm}$.

EU incorporation assay. EU incorporation assays were performed using the Click-iT RNA Alexa Fluor 594 Imaging Kit (Invitrogen) according to the manufacturer's instructions. Briefly, cells were incubated with $1 \mathrm{mM}$ EU for 90 minutes, fixed with $3.7 \%$ PFA for 15 minutes at room temperature, and permeabilized with $0.5 \%$ Triton X-100 for 15 minutes, and Click-iT reaction was performed. DNA was counterstained with Hoechst 33342. Experiments were performed in duplicate, and in each independent experiment, at least 4 different fields and 150 cells were measured per condition. Images were acquired on a Leica AF6000 LX microscope and Hamamatsu digital camera (C4742-80) and analyzed using Image (NIH). Nuclear masks were generated based on Hoechst staining, and mean Alexa Fluor 594 fluorescence intensities per pixel were quantified per nucleus.

NanoString gene expression analysis. Cells were counted in duplicate from cell cultures using FACS (Attune NxT). Pellets of $1 \times 10^{5}, 2 \times 10^{5}$, or $4 \times 10^{5}$ cells were lysed in $40 \mu \mathrm{l}$ Buffer RLT (QIAGEN) for 1 minute at room temperature with strong vortexing and frozen immediately in dry ice. We followed the manufacturer's guidelines for the nCounter cell lysate hybridization and used 2 different gene expression panels: nCounter Human Cancer Reference Kit (GXA-CR1-12) and nCounter CAE Kit (GXA-CAE-12, NanoString Technologies). The signals for each gene were obtained with nSolver software 3.0 (NanoString Technologies).

Immunoprecipitation and Western blot analysis. Protein extract preparation and Western blot analysis were performed as previously described (59). The primary antibodies and the working dilutions are described in Supplemental Table 5. FLAG immunoprecipitation of CDK9-FLAG complexes was performed using ANTI-FLAG M2 Affinity Gel (Sigma-Aldrich) following the manufacturer's guidelines.

Genomic and transcriptomic analysis. ChIP-Seq reads were aligned using Bowtie (http://bowtie-bio.sourceforge.net/index.shtml, v4.3.4) against the human GRCh37/Hhg19 reference genome. Peak calling was performed with Model-based Analysis for ChIP-Seq (MACS, http://liulab.dfci.harvard.edu/MACS/, v1.4.2). Peaks were visualized on the University of California Santa Cruz Genome Browser (https:// genome.ucsc.edu/). Significant cyclin D1-bound intervals were annotated regarding the overlap to genomic features. Sequentially, intervals were annotated as promoter peaks (from $-3,000$ to $+1,000$ around the TSS), then as gene body (from $+1,000$ to transcription termination site [TTS]), further classified as exonic or intronic. Finally, intervals non-overlapping with any of these features were considered as intergenic peaks. Average score profiles were determined using the module Sitepro of the Cis-regulatory Element Annotation System (CEAS, http://liulab.dfci.harvard.edu/CEAS/, v0.9.9.7). SeqMINER v1.3 (http://bips.u-strasbg.fr/seqminer) was studied to visualize ChIP-Seq clusters. Peaks were visualized with the Integrative Genomics Viewer v2.3.81 (https://software.broadinstitute.org/software/igv), and Venn diagrams were created using Venny 2.1.0 (http://bioinfogp.cnb.csic. es/tools/venny/). Pausing ratio for a gene was calculated dividing the number of tags between $(-30,+300)$ around TSS and the number of tags in the gene body (from +300 of TSS to TTS).

The Database for Annotation, Visualization and Integrated Discovery (DAVID) v6.7 (https://david-d.ncifcrf.gov/) application was used to define the functional pathways associated with cyclin D1bound genes. We performed hierarchical cluster analysis using 2 databases (GOTERM_BP_FAT and KEGG_PATHWAY) and the EASE score (modified Fisher's exact $P$ value). The enrichment score was calculated as the geometric mean of -log EASE score, and the percentage of enrichment was calculated as described previously (40), based on the enrichment score of the top hits (enrichment score $>5$ ).

Affymetrix Expression Console software was used to compute robust multichip average (RMA) expression values from $122 \mathrm{MCL}$ patients investigated with Affymetrix Human Genome U133 Plus 2.0 gene expression arrays (Thermo Fisher Scientific) previously deposited in the NCBI's Gene Expression Omnibus database (GEO GSE93291). For each gene, the probe set with the highest average signal was selected for the different analysis. RNA-Seq reads were aligned using Bowtie as described above. To quantify the expression of each gene, we used TopHat v2.0.9 (https://ccb.jhu.edu/software/ tophat) with default parameters and the human GRCh37/Hhg19 as a reference genome. Gene expression values (quantified as reads per kilobase of transcript per million reads [RPKMs]) were calculated using the edgeR Bioconductor package. Analysis of transcription factor binding sites was obtained using ChIP-Seq data from the ENCODE Project in GM12878 (https://www.encodeproject.org/), and data are available through the UCSC Genome Browser.

Statistics. Statistics and graphical results were done in R statistical computing language (v3.1.3) and GraphPad Prism version 4.00 for Windows (GraphPad Software). Data are mainly presented as mean \pm SEM. Differences in mean were analyzed using parametric (paired and unpaired Student's $t$ test and ANOVA) and nonparametric tests (Mann-Whitney, Kolmogorov-Smirnov, and $\chi^{2}$ tests). Correlations were evaluated by calculating Pearson's or Spearman's correlation coefficient or mixed-effects models among replicate measures, when appropriate. Multiple testing was performed by the Holm-Bonferroni method. Two-sided tests were applied throughout, and a $P$ value less than 0.05 (after multiple-testing if required) was considered significant. Statistical tests used are indicated in the figure legends.

Study approval. The study was approved by the Healthcare Ethics Committee of the Hospital Clinic of Barcelona. Written informed consent was obtained from all participants, and the ethics committees approved this consent procedure in accordance with the principles of the Declaration of Helsinki.

\section{Author contributions}

RA, AE, and HSC performed ChIP-Seq and RNA-Seq experiments. SD generated lentiviral silencing models. RA, SD, NG, and CC performed gene expression experiments, Western blotting, and cell culture procedures. Bioinformatics and statistics data analyses were performed by GC, RA, and GC. MP performed NanoString experiments. RA and KK generated the constitutive and inducible models. RA, MS, MLG, and DC performed transcription inhibition experiments. SB and JIMS contributed to discussion and data interpretation. AE, EC, and PJ conceived the study, designed experiments, supervised the project, and interpreted the data. RA, $\mathrm{AE}, \mathrm{SD}$, and PJ wrote the manuscript.

\section{Acknowledgments}

This study was supported by grant PI14/00355, Fondo de Investigaciones Sanitarias-Instituto de Salud Carlos III (to PJ); grant 
SAF2015-64885-R, Ministerio de Economía y Competitividad (to EC); grant AGAUR 2014-SGR-795, Generalitat de Catalunya Suport Grups de Recerca (to EC); grant RD12/0036/0036, the Red Temática de Investigación Cooperativa en Cáncer (RTICC); the European Regional Development Fund "Una manera de fer Europa" CERCA Programme/Generalitat de Catalunya (to EC). EC is an Academia Researcher of the "Institució Catalana de Recerca i Estudis Avançats" (ICREA) of the Generalitat de Catalunya. RA was supported by an FPU fellowship (Ministerio de Educación). SD was supported by a Juan de la Cierva fellowship
(Ministerio de Educación). This work was mainly developed at the Centre Esther Koplowitz (CEK), Barcelona, Spain. We are indebted to the Genomics core facility of the Institut d'Investigacions Biomèdiques August Pi i Sunyer (IDIBAPS) for technical help. We are grateful to A. Davies, A. Girò, L. Plà, M. Sánchez, C. Muro, and N. Villahoz for their excellent technical assistance.

Address correspondence to: Pedro Jares, Anatomic Pathology Department, Hospital Clinic de Barcelona, Villarroel 170, Barcelona, Spain. Phone:34.932275400, ext.2184; Email:pjares@clinic.ub.es.
1. Dickson C, et al. Amplification of chromosome band 11q13 and a role for cyclin D1 in human breast cancer. Cancer Lett. 1995;90(1):43-50.

2. Zukerberg LR, et al. Cyclin D1 (PRAD1) protein expression in breast cancer: approximately onethird of infiltrating mammary carcinomas show overexpression of the cyclin D1 oncogene. Mod Pathol. 1995;8(5):560-567.

3. Jares P, et al. PRAD-1/cyclin D1 gene amplification correlates with messenger RNA overexpression and tumor progression in human laryngeal carcinomas. Cancer Res. 1994;54(17):4813-4817.

4. Callender T, el-Naggar AK, Lee MS, Frankenthaler R, Luna MA, Batsakis JG. PRAD-1 (CCND1)/ cyclin D1 oncogene amplification in primary head and neck squamous cell carcinoma. Cancer. 1994;74(1):152-158.

5. Bosch F, et al. PRAD-1/cyclin D1 gene overexpression in chronic lymphoproliferative disorders: a highly specific marker of mantle cell lymphoma. Blood. 1994;84(8):2726-2732.

6. Pruneri $G$, et al. Immunohistochemical analysis of cyclin D1 shows deregulated expression in multiple myeloma with the $\mathrm{t}(11 ; 14)$. Am J Pathol. 2000;156(5):1505-1513.

7. Benzeno S, et al. Identification of mutations that disrupt phosphorylation-dependent nuclear export of cyclin D1. Oncogene. 2006;25(47):6291-6303.

8. Moreno-Bueno G, et al. Cyclin D1 gene (CCND1) mutations in endometrial cancer. Oncogene. 2003;22(38):6115-6118.

9. Zack TI, et al. Pan-cancer patterns of somatic copy number alteration. Nat Genet. 2013;45(10):1134-1140.

10. Jares P, Colomer D, Campo E. Genetic and molecular pathogenesis of mantle cell lymphoma: perspectives for new targeted therapeutics. Nat Rev Cancer. 2007;7(10):750-762.

11. Coqueret O. Linking cyclins to transcriptional control. Gene. 2002;299(1-2):35-55.

12. Casimiro MC, Velasco-Velázquez M, AguirreAlvarado C, Pestell RG. Overview of cyclins D1 function in cancer and the CDK inhibitor landscape: past and present. Expert Opin Investig Drugs. 2014;23(3):295-304.

13. Pestell RG. New roles of cyclin D1. Am J Pathol. 2013;183(1):3-9.

14. Fu M, et al. Cyclin D1 inhibits peroxisome proliferator-activated receptor gamma-mediated adipogenesis through histone deacetylase recruitment. J Biol Chem. 2005;280(17):16934-16941.

15. Horstmann S, Ferrari S, Klempnauer KH. Regulation of B-Myb activity by cyclin $\mathrm{D} 1$. Oncogene.
2000;19(2):298-306.

16. Reutens AT, et al. Cyclin D1 binds the androgen receptor and regulates hormone-dependent signaling in a p300/CBP-associated factor (P/ CAF)-dependent manner. Mol Endocrinol. 2001;15(5):797-811

17. Zwijsen RM, Wientjens E, Klompmaker R, van der Sman J, Bernards R, Michalides RJ. CDKindependent activation of estrogen receptor by cyclin D1. Cell. 1997;88(3):405-415.

18. Jares P, Campo E. Advances in the understanding of mantle cell lymphoma. Br J Haematol. 2008;142(2):149-165.

19. Gruszka-Westwood AM, et al. Unusual case of leukemic mantle cell lymphoma with amplified CCND1/IGH fusion gene. Genes Chromosomes Cancer. 2002;33(2):206-212.

20. Beà $S$, et al. Uniparental disomies, homozygous deletions, amplifications, and target genes in mantle cell lymphoma revealed by integrative high-resolution whole-genome profiling. Blood. 2009;113(13):3059-3069.

21. Rosenwald A, et al. The proliferation gene expression signature is a quantitative integrator of oncogenic events that predicts survival in mantle cell lymphoma. Cancer Cell. 2003;3(2):185-197.

22. Wiestner A, et al. Point mutations and genomic deletions in CCND1 create stable truncated cyclin D1 mRNAs that are associated with increased proliferation rate and shorter survival. Blood. 2007;109(11):4599-4606.

23. Mohanty A, et al. CCND1 mutations increase protein stability and promote ibrutinib resistance in mantle cell lymphoma. Oncotarget. 2016;7(45):73558-73572.

24. Beà S, et al. Landscape of somatic mutations and clonal evolution in mantle cell lymphoma. Proc Natl Acad Sci U S A. 2013;110(45):18250-18255.

25. Queirós AC, et al. Decoding the DNA methylome of mantle cell lymphoma in the light of the entire B cell lineage. Cancer Cell. 2016;30(5):806-821.

26. Lin CY, et al. Transcriptional amplification in tumor cells with elevated c-Myc. Cell. 2012;151(1):56-67.

27. Nie Z, et al. c-Myc is a universal amplifier of expressed genes in lymphocytes and embryonic stem cells. Cell. 2012;151(1):68-79.

28. Sabo A, et al. Selective transcriptional regulation by Myc in cellular growth control and lymphomagenesis. Nature. 2014;511(7510):488-492.

29. Consortium EP. An integrated encyclopedia of DNA elements in the human genome. Nature. 2012;489(7414):57-74.

30. Loven J, et al. Revisiting global gene expression analysis. Cell. 2012;151(3):476-482.

31. Consortium M. The MicroArray Quality Control (MAQC) project shows inter- and intraplatform reproducibility of gene expression measurements. Nat Biotechnol. 2006;24(9):1151-1161.

32. Yang J, Chen L, Kong X, Huang T, Cai YD. Analysis of tumor suppressor genes based on gene ontology and the KEGG pathway. PLoS One. 2014;9(9):e107202.

33. Xie SQ, Martin S, Guillot PV, Bentley DL, Pombo A. Splicing speckles are not reservoirs of RNA polymerase II, but contain an inactive form, phosphorylated on serine 2 residues of the $\mathrm{C}$-terminal domain. Mol Biol Cell. 2006;17(4):1723-1733.

34. Zeitlinger J, et al. RNA polymerase stalling at developmental control genes in the Drosophila melanogaster embryo. Nat Genet. 2007;39(12):1512-1516.

35. Brookes E, Pombo A. Modifications of RNA polymerase II are pivotal in regulating gene expression states. EMBO Rep. 2009;10(11):1213-1219.

36. Bensaude O. Inhibiting eukaryotic transcription: Which compound to choose? How to evaluate its activity? Transcription. 2011;2(3):103-108.

37. Carter BZ, et al. Triptolide induces caspasedependent cell death mediated via the mitochondrial pathway in leukemic cells. Blood. 2006;108(2):630-637.

38. Wang Y, Lu JJ, He L, Yu Q. Triptolide (TPL) inhibits global transcription by inducing proteasomedependent degradation of RNA polymerase II (Pol II). PLoS One. 2011;6(9):e23993.

39. Bienvenu F, et al. Transcriptional role of cyclin D1 in development revealed by a genetic-proteomic screen. Nature. 2010;463(7279):374-378.

40. Casimiro MC, et al. ChIP sequencing of cyclin D1 reveals a transcriptional role in chromosomal instability in mice. J Clin Invest. 2012;122(3):833-843.

41. Casimiro MC, et al. Kinase-independent role of cyclin D1 in chromosomal instability and mammary tumorigenesis. Oncotarget. 2015;6(11):8525-8538.

42. Walz S, et al. Activation and repression by oncogenic MYC shape tumour-specific gene expression profiles. Nature. 2014;511(7510):483-487.

43. Egloff S, Murphy S. Cracking the RNA polymerase II CTD code. Trends Genet. 2008;24(6):280-288.

44. Chapman RD, Heidemann M, Hintermair C, Eick D. Molecular evolution of the RNA polymerase II CTD. Trends Genet. 2008;24(6):289-296.

45. Rahl PB, et al. c-Myc regulates transcriptional pause release. Cell. 2010;141(3):432-445.

46. Wolf E, Lin CY, Eilers M, Levens DL. Taming of 
the beast: shaping Myc-dependent amplification. Trends Cell Biol. 2015;25(4):241-248.

47. Jirawatnotai S, et al. A function for cyclin D1 in DNA repair uncovered by protein interactome analyses in human cancers. Nature. 2011;474(7350):230-234.

48. Jirawatnotai S, Sittithumcharee G. Paradoxical roles of cyclin D1 in DNA stability. DNA Repair (Amst). 2016;42:56-62.

49. Musgrove EA. Cyclins: roles in mitogenic signaling and oncogenic transformation. Growth Factors. 2006;24(1):13-19.

50. Higashi H, et al. Cyclin-dependent kinase-2 (Cdk2) forms an inactive complex with cyclin D1 since Cdk2 associated with cyclin D1 is not phosphorylated by Cdk7-cyclin-H. Eur J Biochem. 1996;237(2):460-467.

51. Chen R, Chubb S, Cheng T, Hawtin RE, Gand- hi V, Plunkett W. Responses in mantle cell lymphoma cells to SNS-032 depend on the biological context of each cell line. Cancer Res. 2010;70(16):6587-6597.

52. Wang M, et al. Cyclin D1 as a universally expressed mantle cell lymphoma-associated tumor antigen for immunotherapy. Leukemia. 2009;23(7):1320-1328.

53. Bertoli C, Skotheim JM, de Bruin RA. Control of cell cycle transcription during $\mathrm{G} 1$ and $\mathrm{S}$ phases. Nat Rev Mol Cell Biol. 2013;14(8):518-528.

54. Meryet-Figuiere M, et al. Temporal separation of replication and transcription during S-phase progression. Cell Cycle. 2014;13(20):3241-3248.

55. Gaillard H, Herrera-Moyano E, Aguilera A. Transcription-associated genome instability. Chem Rev. 2013;113(11):8638-8661.

56. Mirkin EV, Castro Roa D, Nudler E, Mirkin SM.
Transcription regulatory elements are punctuation marks for DNA replication. Proc Natl Acad SciU S A. 2006;103(19):7276-7281.

57. Morgenstern JP, Land H. Advanced mammalian gene transfer: high titre retroviral vectors with multiple drug selection markers and a complementary helper-free packaging cell line. Nucleic Acids Res. 1990;18(12):3587-3596.

58. Dow EC, Liu H, Rice AP. T-loop phosphorylated Cdk9 localizes to nuclear speckle domains which may serve as sites of active P-TEFb function and exchange between the Brd4 and 7SK/ HEXIM1 regulatory complexes. JCell Physiol. 2010;224(1):84-93.

59. Palomero J, et al. Sox11 promotes tumor angiogenesis through transcriptional regulation of PDGFA in mantle cell lymphoma. Blood. 2014;124(14):2235-2247. 